\title{
Plasma Metabolomic Signatures of Chronic Obstructive Pulmonary Disease and the Impact of Genetic Variants on Phenotype-Driven Modules
}

\author{
Lucas A. Gillenwater, ${ }^{1}$ Katherine A. Pratte, ${ }^{1}$ Brian D. Hobbs, ${ }^{2,3}$ Michael H. Cho, ${ }^{2,3}$ Yonghua Zhuang, ${ }^{4}$ \\ Eitan Halper-Stromberg, ${ }^{5}$ Charmion Cruickshank-Quinn, ${ }^{6}$ Nichole Reisdorph, ${ }^{7}$ Irina Petrache, ${ }^{1,8}$ Wassim W. Labaki, ${ }^{9}$ \\ Wanda K. O'Neal, ${ }^{10}$ Victor E. Ortega, ${ }^{11}$ Dean P. Jones, ${ }^{12}$ Karan Uppal, ${ }^{12}$ Sean Jacobson, ${ }^{1}$ Gregory Michelotti, ${ }^{13}$ \\ Christine H. Wendt, ${ }^{14}$ Katerina J. Kechris, ${ }^{4, \dagger}$ and Russell P. Bowler ${ }^{1,8, *, \dagger}$
}

\begin{abstract}
Background: Small studies have recently suggested that there are specific plasma metabolic signatures in chronic obstructive pulmonary disease (COPD), but there have been no large comprehensive study of metabolomic signatures in COPD that also integrate genetic variants.

Materials and Methods: Fresh frozen plasma from 957 non-Hispanic white subjects in COPDGene was used to quantify 995 metabolites with Metabolon's global metabolomics platform. Metabolite associations with five COPD phenotypes (chronic bronchitis, exacerbation frequency, percent emphysema, postbronchodilator forced expiratory volume at one second $\left[\mathrm{FEV}_{1}\right] /$ forced vital capacity $[\mathrm{FVC}]$, and $\mathrm{FEV} \mathrm{V}_{1}$ percent predicted) were assessed. A metabolome-wide association study was performed to find genetic associations with metabolite levels. Significantly associated single-nucleotide polymorphisms were tested for replication with independent metabolomic platforms and independent cohorts. COPD phenotype-driven modules were identified in network analysis integrated with genetic associations to assess gene-metabolitephenotype interactions.

Results: Of metabolites tested, 147 (14.8\%) were significantly associated with at least 1 COPD phenotype. Associations with airflow obstruction were enriched for diacylglycerols and branched chain amino acids. Genetic associations were observed with 109 (11\%) metabolites, 72 (66\%) of which replicated in an independent cohort. For 20 metabolites, more than $20 \%$ of variance was explained by genetics. A sparse network of COPD phenotypedriven modules was identified, often containing metabolites missed in previous testing. Of the 26 COPD phenotype-driven modules, 6 contained metabolites with significant met-QTLs, although little module variance was explained by genetics.
\end{abstract}

\footnotetext{
${ }^{1}$ National Jewish Health, Denver, Colorado, USA.

${ }^{2}$ Channing Division of Network Medicine, Brigham and Women's Hospital, Boston, Massachusetts, USA.

${ }^{3}$ Division of Pulmonary and Critical Care Medicine, Brigham and Women's Hospital, Boston, Massachusetts, USA.

${ }^{4}$ Department of Biostatistics and Informatics, Colorado School of Public Health, University of Colorado Anschutz Medical Campus, Aurora, Colorado, USA.

${ }^{5}$ Department of Pathology, Johns Hopkins University, Baltimore, Maryland, USA.

${ }^{6}$ Agilent Technologies, Santa Clara, California, USA.

${ }^{7}$ Skaggs School of Pharmacy and Pharmaceutical Sciences, University of Colorado Anschutz Medical Campus, Aurora, Colorado, USA

${ }^{8}$ School of Medicine, University of Colorado, Aurora, Colorado, USA.

${ }^{9}$ Division of Pulmonary and Critical Care Medicine, University of Michigan, Ann Arbor, Michigan, USA.

${ }^{10}$ Lung Institute/Cystic Fibrosis Center, University of North Carolina at Chapel Hill, Chapel Hill, North Carolina, USA.

${ }^{11}$ Department of Internal Medicine, Center for Precision Medicine, Wake Forest School of Medicine, Winston-Salem, North Carolina, USA.

${ }^{12}$ Clinical Biomarkers Laboratory, Division of Pulmonary, Allergy, and Critical Care Medicine, Emory School of Medicine, Atlanta, Georgia, USA.

${ }^{13}$ Metabolon, Inc., Morrisville, North Carolina, USA.

${ }^{14}$ Department of Medicine, University of Minnesota and the VAMC, Minneapolis, Minnesota, USA

†These two authors contributed equally as senior authors.
}

*Address correspondence to: Russell P. Bowler, MD, PhD, National Jewish Health, 1400 Jackson Street, Room K715a, Denver, CO 80206, USA, E-mail: bowlerr@njhealth.org

(c) Lucas A. Gillenwater et al., 2020; Published by Mary Ann Liebert, Inc. This Open Access article is distributed under the terms of the Creative Commons License (http://creativecommons.org/licenses/by/4.0), which permits unrestricted use, distribution, and reproduction in any medium, provided the original work is properly cited. 


\begin{abstract}
Conclusion: A dysregulation of systemic metabolism was predominantly found in COPD phenotypes characterized by airflow obstruction, where we identified robust heritable effects on individual metabolite abundances. However, network analysis, which increased the statistical power to detect associations missed previously in classic regression analyses, revealed that the genetic influence on COPD phenotype-driven metabolomic modules was modest when compared with clinical and environmental factors.
\end{abstract}

Keywords: metabolomics; chronic obstructive pulmonary disease; metabolomic quantitative trait analysis; integrated omics; network analysis

\section{Introduction}

Metabolites are low molecular weight ( $\leq 1500$ Daltons) molecules, representing both endogenous and exogenous (environmentally derived) compounds, which play important roles in signaling, energy expenditure, reproduction, and growth. Metabolites vary greatly across individuals and can act as a unique identifier of an individual through time. ${ }^{1}$ Metabolite expression is thought to be the most proximal signature of health and disease, when compared to other omics (e.g., genomics, transcriptomics, and proteomics). ${ }^{2}$

Recently, there have been several reports suggesting the presence of characteristic metabolic signatures in the blood of individuals with lung diseases such as chronic obstructive pulmonary disease $(\mathrm{COPD})^{3-8}$; however, these reports have typically included only a small number of subjects or a limited annotation of metabolic features $(<500$ metabolites). During the past few years, substantial gains have been made in metabolomics, using analytical chemistry techniques and advanced computational methods to characterize complex biological mixtures. The highly sensitive detection techniques of liquid chromatography tandem mass spectrometry (LC-MS/MS) quantify metabolites from a broad range of classes and are now automated to increase throughput, enabling large cohort-level epidemiological metabolome studies. ${ }^{9,10}$ These strategies have not yet been used on a large scale to study COPD.

COPD is characterized by progressive airflow limitation due to airway and/or alveolar abnormalities and is now the third most common cause of death worldwide, ${ }^{11}$ and is one of the leading causes of medical hospitalizations in the United States. ${ }^{12}$ Cigarette smoking is the greatest environmental risk factor, yet most smokers do not develop clinically important lung disease, and COPD heritability is estimated to be $\sim 37 \% .{ }^{13}$ Furthermore, for those who do develop COPD, there are heterogeneous phenotypes, including emphysema, chronic bronchitis, and frequent COPD exacerbations (Supplementary Fig. S1). ${ }^{14}$ While clinical variables such as age, race, sex, and environmental factors like smoking and body mass index (BMI) have been useful in modeling disease severity, a large amount of unexplained variability in COPD severity remains. ${ }^{15}$ COPD is also associated with increased risk of non-pulmonary diseases independent of smoking history (e.g., cardiovascular disease, osteoporosis, depression, and cancer outside of the lung), suggesting the presence of systemic disturbances in metabolic pathways across comorbidities. ${ }^{16}$ The availability of large longitudinal cohorts for smokers with or at high risk for COPD, such as COPDGene and SPIROMICS, combined with advances in high-throughput metabolomics now permit the large-scale interrogation of the metabolome in COPD.

A similar integrative approach to large-scale transcriptomics ${ }^{17}$ and proteomics ${ }^{18}$ studies in COPDGene, SPIROMICS, and other cohorts has revealed that a significant amount of variation in many biomarkers is explained by genetic variation, which may also impact metabolome signatures, as recently reported. ${ }^{19}$ In addition, genome-wide association studies (GWASs) have found multiple genetic loci associated with COPD. ${ }^{20}$ Thus, it is important to consider the role of genetic background in assessing how the metabolome relates to COPD; however, to our knowledge, comprehensive studies integrating genetics with comprehensive metabolomic profiling in COPD are lacking. This study identifies plasma metabolites associated with COPD-related phenotypes and addresses the impact of genetic variation on metabolomic profiles in COPD.

\section{Materials and Methods}

Study populations

Discovery. The NIH-sponsored multicenter Genetic Epidemiology of COPD (COPDGene; ClinicalTrials .gov Identifier: NCT01969344) study was approved and reviewed by the institutional review board at all participating centers. ${ }^{21}$ All study participants provided written informed consent. This study enrolled 10,198 non-Hispanic white (NHW) and African American 
(AA) individuals from January 2008 until April 2011 (Phase 1 ), who were $45-80$ years of age with $\geq 10$ packyear smoking history and no exacerbation for $>30$ days. In addition, 465 age- and gender-matched healthy individuals with no history of smoking were enrolled as controls (mostly at Phase 2). From July 2013 to July 2017, 5697 subjects returned for an in-person 5-year visit. Each in-person visit included spirometry before and after albuterol, quantitative computed tomography (CT) imaging of the chest, and blood sampling.

From two clinical centers (National Jewish Health and University of Iowa), 1136 subjects (1040 NHW, 96 AA) participated in an ancillary study in which they provided fresh frozen plasma collected using an $8.5 \mathrm{~mL}$ p100 tube (Becton Dickinson) at Phase 2. After excluding AA subjects due to small sample size and subjects lacking genotype data, to avoid confounding genetic associations due to ancestry, 957 subjects comprised the Discovery cohort (Supplementary Fig. S2).

COPDGene: Emory. From the Discovery cohort, 271 COPDGene NHW subjects who previously had their metabolome quantified at Phase 2 on a separate platform were used as a technical COPDGene-Emory cohort. This cohort will be referred to as COPDGeneEmory.

SPIROMICS: Metabolon/UC. Two cohorts from the Subpopulations and Intermediate Outcome Measures in COPD study (SPIROMICS) (ClinicalTrials.gov Identifier: NCT01969344) were used for replication. ${ }^{22}$ The SPIROMICS-Metabolon and SPIROMICS-UC subjects consisted of 445 and 76 NHW subjects, respectively, who provided fresh frozen plasma using a $10 \mathrm{~mL}$ EDTA tube (Becton Dickinson) before a research bronchoscopy. ${ }^{23}$

\section{Clinical data and definitions}

COPD was defined using spirometric evidence of airflow obstruction (post-bronchodilator forced expiratory volume at one second $\left[\mathrm{FEV}_{1}\right] /$ forced vital capacity [FVC] <0.70). PRISm subjects had an $\mathrm{FEV}_{1}$ percent predicted $\left(\mathrm{FEV}_{1} \mathrm{pp}\right)<80 \%$ with an $\mathrm{FEV}_{1} / \mathrm{FVC} \geq 0.7$. PRISm subjects have recently been recognized as having a higher prevalence of symptoms and worse outcomes compared to traditionally defined controls, ${ }^{24}$ and were thus included in all cohorts. Chronic bronchitis was defined as self-reported chronic cough and sputum for at least 3 months in each of the 2 years before Phase 2. Percent emphysema was quantified by percent of lung voxels less than -950 Hounsfield Units (\% low attenuation areas) on the inspiratory CT scans. Visual emphysema was assessed as previously described. ${ }^{25}$ Exacerbations were defined as acute worsening of respiratory symptoms requiring treatment with oral corticosteroids and/or antibiotics, emergency room visit, or hospital admission. ${ }^{26}$

\section{Metabolite profiling}

Discovery platform. P100 plasma was profiled using the Metabolon (Durham) global metabolomics platform, as described. ${ }^{27-29}$ Briefly, samples were extracted with methanol under vigorous shaking for $2 \mathrm{~min}$ (Glen Mills GenoGrinder 2000) followed by centrifugation to remove protein, dissociate small molecules bound to protein or trapped in the precipitated protein matrix, and recover chemically diverse metabolites. The resulting extract was divided into five fractions: two for analysis by two separate reverse-phase/ultrahighperformance liquid chromatography/tandem mass spectrometry (RP/UPLC-MS/MS) methods with positive ion mode electrospray ionization (ESI), one for analysis by RP/UPLC-MS/MS with negative ion mode ESI, one for analysis by hydrophilic interaction liquid chromatography (HILIC)/UPLC-MS/MS with negative ion mode ESI, and one was reserved for backup.

Metabolon has developed peak detection and integration software to generate a list of (mass-to-charge) $\mathrm{m} / \mathrm{z}$ ratios, retention indices (RI), and area under the curve values for each detected metabolite, as described in detail. $^{27-29}$ User-specified criteria for peak detection included thresholds for signal to noise ratio, area, and width. Relative standard deviations of peak area were determined for internal and recovery standards to confirm extraction efficiency, instrument performance, column integrity, chromatography, and mass calibration.

The biological data sets, including quality control samples, were chromatographically aligned based on a retention index that utilized internal standards assigned a fixed RI value. The RI of the experimental peak was determined by assuming a linear fit between flanking RI markers whose RI values are set. Peaks were matched against an in-house library of authentic standards and routinely detected unknown compounds specific to the respective method. Identifications were based on retention index values, experimental precursor mass match to the library authentic standard within $10 \mathrm{ppm}$, and quality of MS/MS match. All proposed identifications were then manually reviewed and curated by an analyst who approved or rejected each 
identification based on the criteria above. The platform reported 1392 features, including 1064 annotated features, which were grouped by Metabolon into "super pathways," including 436 lipids, 261 xenobiotics, 207 amino acids, 40 peptides, 38 cofactors and enzymes, 35 nucleotides, 25 carbohydrates, 11 energy pathway compounds, and 11 partially characterized molecules (Supplementary Table S1). All compounds are further annotated by "subpathway" (e.g., "sphingomyelins," "carnitine metabolism," and "lysine metabolism").

COPDGene: Emory. Compounds from p100 fresh frozen plasma were extracted using an untargeted LCMS-based metabolomic quantification protocol from the laboratory of Dean Jones at Emory University as described previously. ${ }^{30}$ In brief, eight stable isotope internal standards in $130 \mu \mathrm{L}$ acetonitrile were mixed with $65 \mu \mathrm{L}$ of plasma. Samples were precipitated and chromatographic separation of the supernatant was performed on a Dionex Ultimate 3000 UHPLC with a dual column compartment for column switching. Reverse phase (C18), anion exchange (AE), and HILIC preceded mass spectral detection using a Thermo Scientific Q-Exactive HF mass spectrometer in continuous full scan mode at 70,000 resolution (scan range $85-1275 \mathrm{~m} / \mathrm{z}$ for all analyses other than AE, AE scan range was $100-1500 \mathrm{~m} / \mathrm{z}$ ).

Data were extracted using xMSanalyzer ${ }^{31}$ and annotated using xMSannotator. ${ }^{32}$ There were 4474 features identified among the 271 samples.

SPIROMICS: Metabolon. P100 plasma was profiled using the Metabolon Global Metabolomics Platform, as described for the Discovery cohort. The platform reported 1174 features (unannotated features were excluded) with a super pathway breakdown of 435 lipids, 228 amino acids, 318 xenobiotics, 43 cofactors and vitamins, 43 peptides, 41 nucleotides, 30 partially characterized molecules, 25 carbohydrates, and 11 energy metabolites.

SPIROMICS: UC. Samples from p100 fresh frozen plasma underwent LC-MS profiling in the laboratory of Nichole Reisdorph at the University of Colorado Anschutz Medical Campus as previously described. ${ }^{33,34}$ In brief, cold methanol was added to plasma sample aliquots containing internal standards to precipitate proteins. Supernatants were extracted using liquidliquid extraction with methyl tert-butyl ether to obtain a lipid fraction and a small molecule aqueous fraction. Samples were analyzed in positive mode using C18 and HILIC on an Agilent 6545 quadrupole time-of- flight (QTOF) and 6520 QTOF, respectively. Spectral peaks were extracted using MassHunter Profinder B.08 (Agilent). Features were annotated using Mass Profiler Professional (Agilent) using either an in-house accurate mass and retention time (AMRT) database or exact mass and isotope ratios for the compounds that were not in the AMRT database. There were 10,561 features detected among the 81 samples.

\section{Genotyping}

Discovery and COPDGene: Emory. Subjects were genotyped using the HumanOmniExpress array (Illumina) employing BeadStudio quality control, which included reclustering on project samples following Illumina guidelines, as previously described for COPDGene. Genotype imputation was performed using the Michigan Imputation Server and the HRC 1.1 reference NHW and the 1000 Genome Phase $1 \mathrm{v} 3$ for AAs. ${ }^{35}$ Ancestry-based principal components (PCs) were calculated and used as previously described. ${ }^{36,37}$ Variants were filtered to include only single-nucleotide polymorphisms (SNPs) with minor allele frequencies $>1 \%$ in the sample population.

SPIROMICS. Subjects were genotyped using the HumanOmniExpress array (Illumina) as previously described. ${ }^{38}$ Around 683,998 directly genotyped SNPs passed quality control after the removal of SNPs significantly deviating from Hardy-Weinberg expectations $(p<0.0001)$, missing allele data (any “0”), and with a genotype call rate $<90 \%$ and heterozygous haploid genotypes. Genotype imputation was performed using the Michigan imputation server and the HRC 1.1 reference NHW ancestry-based PCs were calculated and used as previously described. ${ }^{36}$ Variants were filtered to include only SNPs with minor allele frequencies $>1 \%$ in the sample population.

\section{Statistical analysis}

Data sets and availability. Clinical data and genotype data can be found on $\mathrm{dbGaP}$ for COPDGene (phs000179.v6.p2) and SPIROMICS (phs001119.v1.p1). For COPDGene, the following dataset was used: COPDGene_P1P2_All_Visit_29Sep2018. For SPIROMICS, the CORE 5 data sets were used. Discovery metabolomic data are available at the NIH Common Fund's National Metabolomics Data Repository website, the Metabolomics Workbench, https://www.metab olomicsworkbench.org where it has been assigned project ID PR000907. 
Pre-analyses

Discovery and SPIROMICS: Metabolon. Unless otherwise mentioned, all metabolite data processing and analysis were performed in $\mathrm{R}$ (v3.5.1). A data normalization step was performed to correct variation resulting from instrument interday tuning differences: metabolite intensities were divided by the metabolite run day median and then multiplied by the overall metabolite median. It was determined that no further normalization was necessary based on the reduction in the significance of association between the top metabolomics PCs (calculated using the R function "prcomp") and sample run day after normalization (Supplementary Fig. S3). Metabolites were excluded if $>20 \%$ of samples were missing values. ${ }^{39}$ For the 995 remaining metabolites, missing values were imputed across metabolites with $k$-nearest neighbor imputation $(k=10)$ using the R package "impute." 40

To detect and remove outliers, median standard deviation scores ( $z$-scores) were calculated across metabolites at the subject level. Subjects with aggregate metabolite median $z$-scores $>3.5$ standard deviation from the mean $(N=6)$ of the cohort were removed (Supplementary Fig. S4). All measured metabolite relative abundances were transformed using the normal quantile transformation, as this type of rank-based transformation can remove possible bias due to outliers or skewed distribution. ${ }^{41}$

COPDGene: Emory. Metabolite data were preprocessed using the MSPrep R package. ${ }^{42}$ Data were first imported and summarized across three technical replicates before filtering to include only compounds with $<20 \%$ missingness over samples. This reduced the data to 2891 compounds, 163 of which were annotated with compound name. Bayesian principal component analysis was employed for imputation ${ }^{43,44}$ of missing values before ComBat batch correction and quantile normal transformation. ${ }^{45}$

SPIROMICS. Metabolite data were pre-processed using the MSPrep R package ${ }^{42}$ as described previously. ${ }^{33}$ Raw data were filtered to include only compounds with $<20 \%$ missingness over samples. This reduced the data to 7918 compounds, 3843 of which were annotated by compound name. $k$-Nearest neighbor imputation $(k=5)$ was employed for imputation of missing values before ComBat batch correction and quantile normal transformation ${ }^{45}$

Exploring associations between COPD and metabolites in Discovery cohort. Phenotype-metabolite associa- tions were tested using various regression models and covariates based on previous literature (Supplementary Table S2 ${ }^{46}$ for five phenotypes. Significance was determined within each phenotype at a $p$-value $<5.03 \times$ $10^{-5}$ after employing a Bonferroni correction to account for multiple testing over 995 metabolites.

Metabolome-wide association study. First, the additive effects of SNPs on metabolite abundances were assessed in the Discovery cohort with linear regression using the R package "MatrixEQTL" (version 2.2). ${ }^{47}$ Models were adjusted for clinical covariates (clinical center, sex, age, BMI, smoking pack years, and current smoking status) as well as ancestry-based PCs and as previously described. ${ }^{36}$ Metabolite quantitative trait loci (met-QTLs) were considered significant at $p$-value $<6.6 \times 10^{-12}$ for genome-wide significance after employing a Bonferroni correction to account for multiple testing across 995 metabolites and 7,641,295 genotyped and imputed SNPs.

Metabolome-wide association study replication across metabolomic platforms and cohorts. Significant metQTL SNPs were tested for associations in the COPDGene-Emory and SPIROMICS replication platforms, using the same methods as previously described for the Discovery cohort and the Bonferroni correction for multiple testing.

Recursive conditioning. If $K$ met-QTL-SNPs were associated with a metabolite with $p$-values smaller than $6.6 \times 10^{-12}, p$-values were calculated for each of the $K-1$ SNPs conditioning on the top SNP identified in the met-QTL analysis and other covariates (age, sex, BMI, smoking status, smoking pack-years, and clinical center). The SNP with the smallest $p$-value was considered an independent met-QTL if $p$-value $<0.05 /(K-1)$, where $0.05 /(K-1)$ was the $p$-value threshold by Bonferroni correction. We applied this procedure iteratively until the smallest $p$-value was larger than $0.05 / T$, where $T$ is the number of remaining SNPs. ${ }^{36}$

Exploring met-QTLs. Percent variance explained by SNPs and clinical variables was calculated using the coefficient of determination $\left(r^{2}\right)$. met-QTL features were characterized using the "-most_severe_variant" filter and nearest genes were identified using the "-nearest symbol" argument in the Ensembl Variant Effect Predictor (VEP) tool (V97). ${ }^{48}$

Enrichment analysis. Group enrichment (i.e., subpathway for metabolites or variant class for SNPs) 
among significantly associated features was statistically assessed against the entire feature set using a one-tailed Fisher's exact test. ${ }^{49}$ Results were adjusted using Benjamini and Hochberg ${ }^{50}$ (a.k.a. false discovery rate) with an alpha of 0.05 .

Network analysis of metabolic interaction. As metabolic pathway annotations are arbitrarily defined and ignore unannotated compounds, ${ }^{51,52}$ we sought to identify COPD-affected pathways in a strictly data-driven manner. This was performed in a two-step procedure. First, we generated a Gaussian graphical model (GGM) of metabolite co-abundance based on partial correlation coefficients corrected for the effects of all other metabolites and potential confounders (age, sex, BMI, smoking status, smoking pack-years, and clinical center). ${ }^{53}$

The use of partial coefficients in the GGM model seeks to overcome a major drawback of other correlation networks (e.g., Pearson's) by conditioning against correlations with all other variables. Edges between metabolites were present if partial correlations were statistically significant at an alpha of 0.05, after Bonferroni correcting for $\left(\begin{array}{c}995 \\ 2\end{array}\right)$ tests, with a positive partial correlation $>0.2$ to declare whether an edge is "present" in the network view.

Negative partial correlations likely represent spurious signals as detailed in previous publications, ${ }^{53,54}$ and thus were removed. To infer potential genetic effects, results from the metabolome-wide association study (mWAS) were included in the network view by introducing "SNP" nodes with edges present between met-QTLs and associated metabolites. ${ }^{54}$ In summary, the combined GGM and mWAS approach will provide an unbiased map of metabolic pathways and their genetic influences. ${ }^{53}$

The first step based on the GGM identifies partially correlated metabolites, but does not consider phenotypes. Therefore, in the second step, metabolomic modules associated with COPD phenotypes were identified using a greedy search algorithm. ${ }^{55}$ Each phenotype was tested separately. Briefly, each metabolite node was regressed against the phenotype and scored using the negative logarithmized $p$-value of the phenotype beta coefficient. Phenotypes were adjusted for the same covariates as identified in previous literature (Supplementary Table S2) ${ }^{46}$ by regressing the phenotype against those covariates and using the residuals as the independent variable in the model. Next, starting with a seed node, each neighboring node is added iteratively to the candi- date module by averaging metabolite intensities, and this extended module is scored by linear regression as previously described. The neighbor is added only if the score of the newly extended module is higher than the scores of all the single components. Any overlapping optimal module is combined in a final step into a single module and scored by the scoring function, using the same rules as before to determine inclusion.

In summary, this approach systematically identifies phenotype-affected modules based on a GGM-derived network of metabolic pathways. Both steps were performed using the R package "MoDentify" ized using Cytoscape (v3.71). ${ }^{56}$

\section{Results}

Metabolome data substructure

Before reducing data by the exclusion criteria, we first explored the metabolomic profiles of all COPDGene subjects with metabolomes quantified by Metabolon at Phase 2. These subjects were representative of all COPDGene subjects who returned for the 5-year follow-up (Supplementary Table S3). Pairwise correlations among metabolites were assessed using Pearson's $r$ for hierarchical clustering within Metabolon-defined super pathways. Beyond a positively correlated cluster of lipids, metabolites exhibited minor correlation (Supplementary Fig. S5).

Univariate demographic associations were then assessed with linear regression models. The demographic variables most strongly associated with metabolites included age, sex, race, and smoking status (Supplementary Table S4 and Supplementary Fig. S5). Of the 995 metabolites tested, $398(40.2 \%)$ were significantly associated with age, 319 (32.1\%) with sex, 355 (37.2\%) with race, 250 with BMI (25.1\%), and 128 (12.9\%) with smoking status. Enrichment analysis found androgenic steroids, acylcarnitines, and dicarboxylates to be enriched for associations with age; sphingomyelin, androgenic steroids, and phosphatidylcholines with sex; xanthines and dicarboxylates with race; and diacylglycerols and branched chain amino acids (BCAAs) for BMI (Supplementary Table S5).

\section{Study subjects}

Demographic and clinical characteristics of the Discovery cohort are shown in Table 1 . There were significant differences between PRISm subjects, current or former smoker controls, and COPD across age, sex, BMI, smoking status, and smoking pack-years. Among the met-QTL replication cohorts, COPDGene-Emory subjects were representative of the Discovery cohort, 
Table 1. Demographics of Discovery cohort

\begin{tabular}{|c|c|c|c|c|c|c|}
\hline & Total & PRISm & Control & COPD & Missing & $p$ \\
\hline No. of participants (\%) & 957 & $85(8.9)$ & $390(40.8)$ & 468 (48.9) & $14(1.4)$ & \\
\hline $\mathrm{Age}^{\mathrm{a}}$ & $68.3(8.4)$ & $66.7(7.3)$ & $65.9(8.5)$ & $70.5(8.0)$ & $70.7(5.8)$ & $<0.0001$ \\
\hline Male sex (\%) & $490(51.2)$ & $31(36.5)$ & $184(47.2)$ & $268(57.3)$ & $7(50.0)$ & 0.0002 \\
\hline BMI (\%) & $29.1(6.2)$ & $32.6(7.7)$ & $29.3(5.6)$ & $28.2(6.1)$ & $27.9(5.3)$ & $<0.0001$ \\
\hline Current smoker (\%) & $204(21.3)$ & $24(28.2)$ & $78(20.0)$ & $98(20.9)$ & $4(28.6)$ & 0.1914 \\
\hline Smoking pack-years ${ }^{\mathrm{a}}$ & $46.0(24.9)$ & $48.6(24.3)$ & $36.1(19.5)$ & $53.6(26.2)$ & $51.7(22.2)$ & $<0.001$ \\
\hline $\mathrm{FEV}_{1}{\mathrm{pp} \_u t a \mathrm{~h}^{\mathrm{a}}}^{\mathrm{a}}$ & $76.6(26.5)$ & $70.2(7.4)$ & $99.2(11.5)$ & $58.9(23.5)$ & NA & NA \\
\hline $\mathrm{FEV}_{1} / \mathrm{FVC}^{\mathrm{a}}$ & $0.7(0.2)$ & $0.8(0.0)$ & $0.8(0.0)$ & $0.5(0.1)$ & NA & NA \\
\hline Percent emphysema ${ }^{a}$ & $7.3(10.2)$ & $1.6(2.5)$ & $2.2(2.6)$ & $12.9(12.2)$ & $9.2(11.7)$ & $<0.0001$ \\
\hline
\end{tabular}

Chi-square tests were used to test for differences between groups in binary variables. One-way ANOVA tests were performed to test for differences between groups in continuous variables.

PRISm, Preserved Ratio Impaired Spirometry ${ }^{23}$; COPD is defined by GOLD score $\geq 1$; missing, 14 subjects were deemed ineligible for spirometry and thus did not have a defined GOLD status. These subjects were still included in analyses with other COPD phenotypes and the met-QTL analysis.

$\mathrm{BMI}$, body mass index $\left(\mathrm{kg} / \mathrm{m}^{2}\right) ; \mathrm{FEV}_{1} / \mathrm{FVC}$, post-bronchodilator forced expiratory volume at one second/forced vital capacity; $\mathrm{FEV}_{1} \mathrm{pp} \mathrm{FEV}_{1}$ percent predicted.

${ }^{\mathrm{a}}$ Mean and standard deviations provided.

COPD, chronic obstructive pulmonary disease.

while the SPIROMICS subjects were slightly younger and healthier, as evidenced by the higher $\mathrm{FEV}_{1} \mathrm{pp}$ and lower percent emphysema (Table 2).

\section{Metabolites associated with COPD phenotypes \\ in the Discovery cohort}

Of the 995 metabolites tested for associations, 147 (14.8\%) were significantly associated with at least 1 of the 5 COPD phenotypes studied (Fig. 1A, full results in Supplementary Tables S6-S10). There was no metabolite significantly associated with chronic bronchitis. For exacerbations and emphysema, only one metabolite was identified in each. Higher abundance of $\mathrm{N}, \mathrm{N}, \mathrm{N}$-trimethyl-alanylproline betaine (TMAP) was significantly associated with a decrease in exacerbation frequency $\left(p=3.75 \times 10^{-5}\right)$, while increased abundance in a tricarboxylic cycle metabolite (citrate) was significantly associated with higher percent emphysema $\left(p=5.2 \times 10^{-6}\right)$.

For the COPD phenotypes characterized by airflow obstruction, 145 metabolites from 55 subpathways were sig- nificantly associated with either $\mathrm{FEV}_{1}$ pp or $\mathrm{FEV}_{1} / \mathrm{FVC}$. For $\mathrm{FEV}_{1} / \mathrm{FVC}$, there were significant associations with 99 metabolites from 30 subclasses, 39 (39.4\%) of which were positively associated (Fig. 1B). Glycophosphatidylinositol (Fig. 1C), propionylcarnitine (C3), and ergothioneine, a xenobiotic (Fig. 1E), were most strongly associated $\left(p=2.57 \times 10^{-13}, 4.8 \times 10^{-13}\right.$, and $4.1 \times 10^{-11}$, respectively). Enrichment analysis found metabolites in the diacylglycerol and BCAA (leucine, isoleucine, and valine) subpathways to be enriched for associations with $\mathrm{FEV}_{1} / \mathrm{FVC}$ (Supplementary Table S11). For FEV ${ }_{1} \mathrm{pp}, 79$ metabolites from 23 subclasses were significantly associated, with lipid phosphocholine (Fig. 1D), ergothioneine, and carbohydrate N6-carboxymethyllysine most significantly associated $\left(p=3.3 \times 10^{-13}, 3.28 \times 10^{-12}\right.$, and $1.4 \times 10^{-11}$, respectively).

\section{Identification of SNPs associated with metabolites}

We next investigated the genetic contribution to metabolite abundances by investigating the relationship between

Table 2. Demographics of replication cohorts

\begin{tabular}{|c|c|c|c|c|}
\hline & COPDGene-Emory & SPIROMICS-Metabolon & SPIROMICS-UC & $p$ \\
\hline No. of participants & 271 & 445 & 76 & NA \\
\hline $\mathrm{Age}^{\mathrm{a}}$ & $67.3(8.4)$ & $65.3(8)$ & $61.6(8)$ & $<0.001$ \\
\hline Male sex (\%) & $127(46.9)$ & $244(54.8)$ & $40(52.6)$ & 0.1164 \\
\hline BMI (\%) & $28.7(5.8)$ & $28.1(4.9)$ & $28.3(4.8)$ & 0.3675 \\
\hline Current smoker (\%) & $57(21.0)$ & $116(26.4)$ & $19(25.7)$ & 0.2665 \\
\hline Smoking pack-years ${ }^{\mathrm{a}}$ & $43.9(23.3)$ & $47.8(31.6)$ & $43.2(24)$ & 0.1362 \\
\hline $\mathrm{FEV}_{1} \mathrm{pp}_{\text {-utah }}{ }^{\mathrm{a}}$ & $77.1(25.3)$ & $79.4(23.5)$ & $89.3(20.8)$ & 0.0004 \\
\hline $\mathrm{FEV}_{1} / \mathrm{FVC}^{\mathrm{a}}$ & $0.7(0.1)$ & $0.6(0.1)$ & $0.7(0.1)$ & 0.0015 \\
\hline Percent emphysema ${ }^{a}$ & $6.9(9.7)$ & $6.1(8.2)$ & $4(4.8)$ & 0.038 \\
\hline
\end{tabular}

Chi-square tests were used to test for differences between groups in binary variables. One-way ANOVA tests were performed to test for differences between groups in continuous variables.

${ }^{a}$ Mean and standard deviation provided unless otherwise noted. 

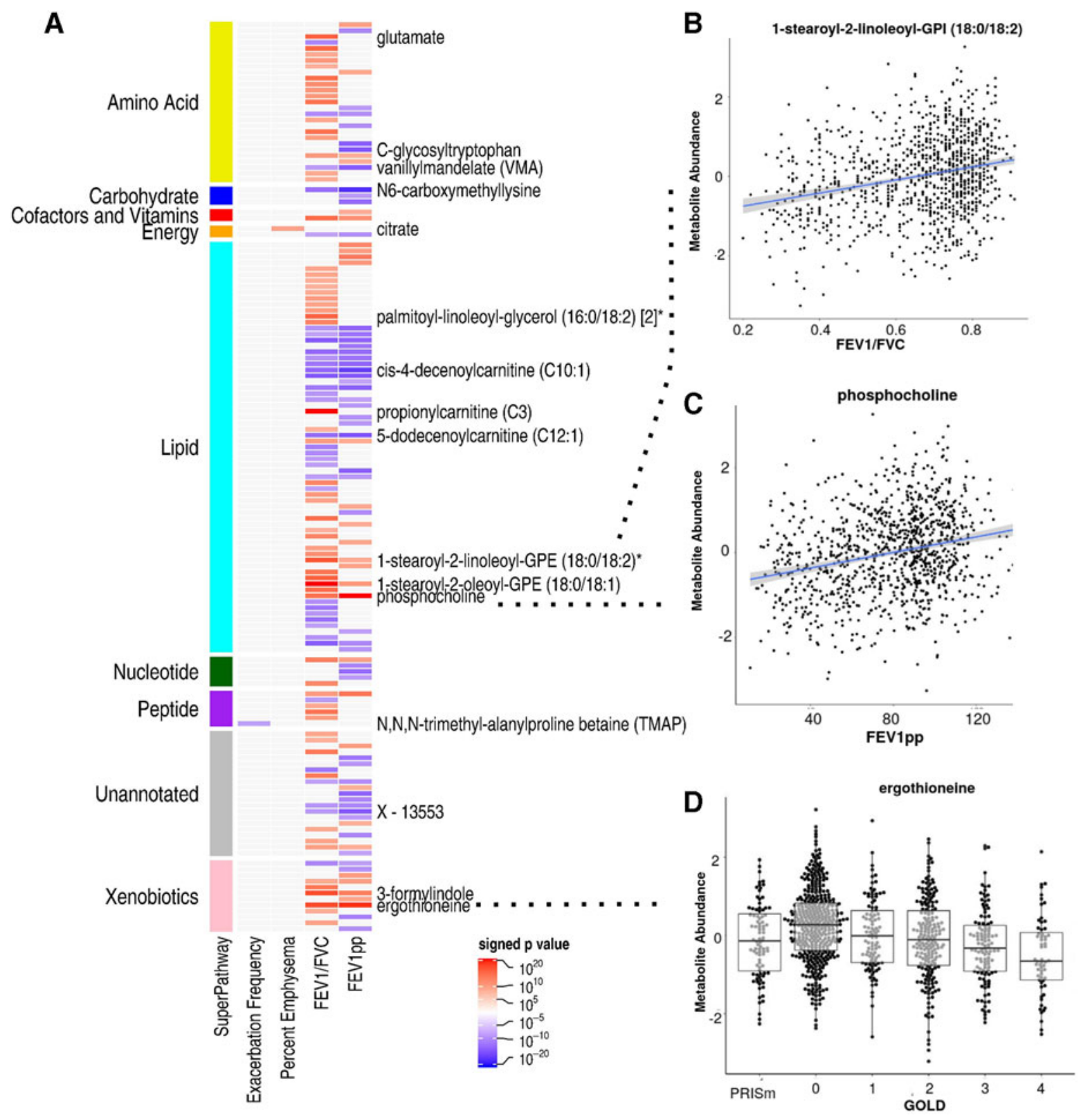

FIG. 1. Metabolite associations with COPD. (A) Heat map of signed $p$-values. Metabolites are organized by super pathway. Red intensity indicates positive direction of effect, while blue intensity indicates negative. Only select metabolites most significantly associated are labeled. (B) Scatter plot of 1-stearoyl-2-linoleoyl-GPI (18:0/ 18:2) abundance by $F E V_{1} / F V C$ ratio. (C) Scatter plot of phosphocholine abundance by FEV 1 pp. (D) Bee swarm of ergothioneine abundance by GOLD stage. Ergothioneine was one of the topmost associated metabolites with all airflow obstruction phenotypes. Metabolites are color coded by Super Pathway designation. Metabolite abundances are inverse normal transformed. *Indicates compounds that have not been officially confirmed based on a standard, but Metabolon is confident in its identity. COPD, chronic obstructive pulmonary disease; $\mathrm{FEV}_{1}$, forced expiratory volume at one second; $\mathrm{FEV}_{1} \mathrm{pp}$, FEV 1 percent predicted; FVC, forced vital capacity; GPI, glycophosphatidylinositol. 
genotypes and metabolites. Of the $\sim 7.6$ million genotyped and imputed SNPs tested, we identified 4281 met-QTL SNPs associated with 109 (10.95\%) of metabolites tested in the Discovery cohort (Fig. 2A and Supplementary Table S12). An interactive plot displaying met-QTL SNP association with metabolite subclass can be found at https://plot.ly/ lagillenwater/7 Using recursive conditioning, 79 independent SNPs were identified with an additive relationship with the 109 metabolites (Supplementary Table S13 and Fig. 2C, D). At least $15 \%$ of the variance in 20 metabolites was explained by 1 or more of these SNPs, often much more than observed in clinical variables (Table 3 and Fig. 2E).

The strongest genetic link was between a missense variant in the PYROXD2 region of chromosome 10, rs2147896, which explained $50.48 \%$ of the variance of N6-methyllysine; in contrast, the clinical variables explained only $0.64 \%$ of the variance in this metabolite (Fig. 2E). For 13 metabolites, 2 or more independent met-QTL SNPs contribute to metabolite variance (Table 3). For example, $58.90 \%$ of variance in N2acteyl, N6-methyllysine is explained by variants in PYROXD2 (34.26\%) and NAT8 (24.64\%) regions.

\section{Biologic significance of met-QTL SNPs}

and associated metabolites

Next, we set out to determine if these met-QTL SNPs have been previously associated with COPD, lung function, or metabolite levels. First, we cross-referenced the 4281 variants with 279 significant SNPs from a recent lung function GWAS ${ }^{57}$ and164 reported primary and secondary COPD GWAS SNPs ${ }^{58}$ for overlapping associations. Next, we compared the met-QTL variants with published associations in the NHGRI GWAS catalog. $^{59}$ Of the expanded variant set, 351 SNPs have been previously reported, mostly in other metabolomic analyses. $^{54,60-63}$ There were seven met-QTL SNPs that had previously been associated with smoking habits (SNPs rs10254729, rs10469966, rs12825376, rs13437771, rs2072113, rs2421667, and rs883403), although no met-QTL SNP overlapped with lung function or COPD GWAS SNPs.

Using Ensembl VEP, we found intronic SNPs to be the most represented met-QTL SNP class (64.5\%), followed by intergenic variants (12.6\%) (Supplementary Table S14 and Fig. 3A). Intronic variants were also the most significantly enriched, followed by $3^{\prime}$ untranslated region and missense variants $\left(q=3.48 \times 10^{-79}\right.$, $3.99 \times 10^{-33}$, and $\left.2.68 \times 10^{-25}\right)$. At least $50 \%$ of metabolites in 13 subpathways had met-QTLs, with all 3 of the metabolites in the hemoglobin and porphyrin metabolism subpathway having significant genetic associations (Supplementary Table S15 and Fig. 3B). Although metabolites with met-QTLs were not enriched for any subpathway, at the super-pathway level, an enrichment of amino acids was found $(q=0.048)$.

\section{Replication of met-QTL SNPS}

We used three strategies to test for replication of metQTL SNPs (see Materials and Methods section). First, we used an independent high-resolution LC-MS strategy in a different laboratory in the same cohort (COPDGene-Emory) (Supplementary Table S16). Second, we used an independent cohort with data also quantified by Metabolon (SPIROMICSMetabolon) (Supplementary Table S17). Third, we used an independent cohort with data from an independent platform (SPIROMICS-UC) (Supplementary Table S18). The cohort with the greatest replication was SPIROMICS-Metabolon where 72 met-QTL associations replicated (Fig. 4, Table 4, and Supplementary

FIG. 2. Genome-wide associations between SNPs and metabolites. (A) Discovery mWAS Manhattan plot showing $-\log 10 p$-values from mWAS tests. The blue and red dashed lines indicate false discovery rate and Bonferroni significance, respectively. Loci in which $>20 \%$ of the metabolite variance is explained by a single SNP are labeled by nearest gene and metabolites affected. Metabolite text colors coded by Super Pathway. (B-D) Bee swarms of inverse normal transformed metabolite abundances by genotype for metabolites of different subpathways with the greatest variance explained by one SNP. Overlayed box plots represent the median and interquartile range of transformed metabolite abundance. (E) Bar plot of the percent variation for metabolites explained by clinical (green), top mQTL SNP (blue), the second independent mQTL SNP (orange), and any more independent mQTL SNPs (red). The gray indicates unknown variance. Clinical factors include age, sex, BMI, smoking status, smoking pack-years, and clinical center. BMI, body mass index; mWAS, metabolome-wide association study; SNPs, singlenucleotide polymorphisms. 


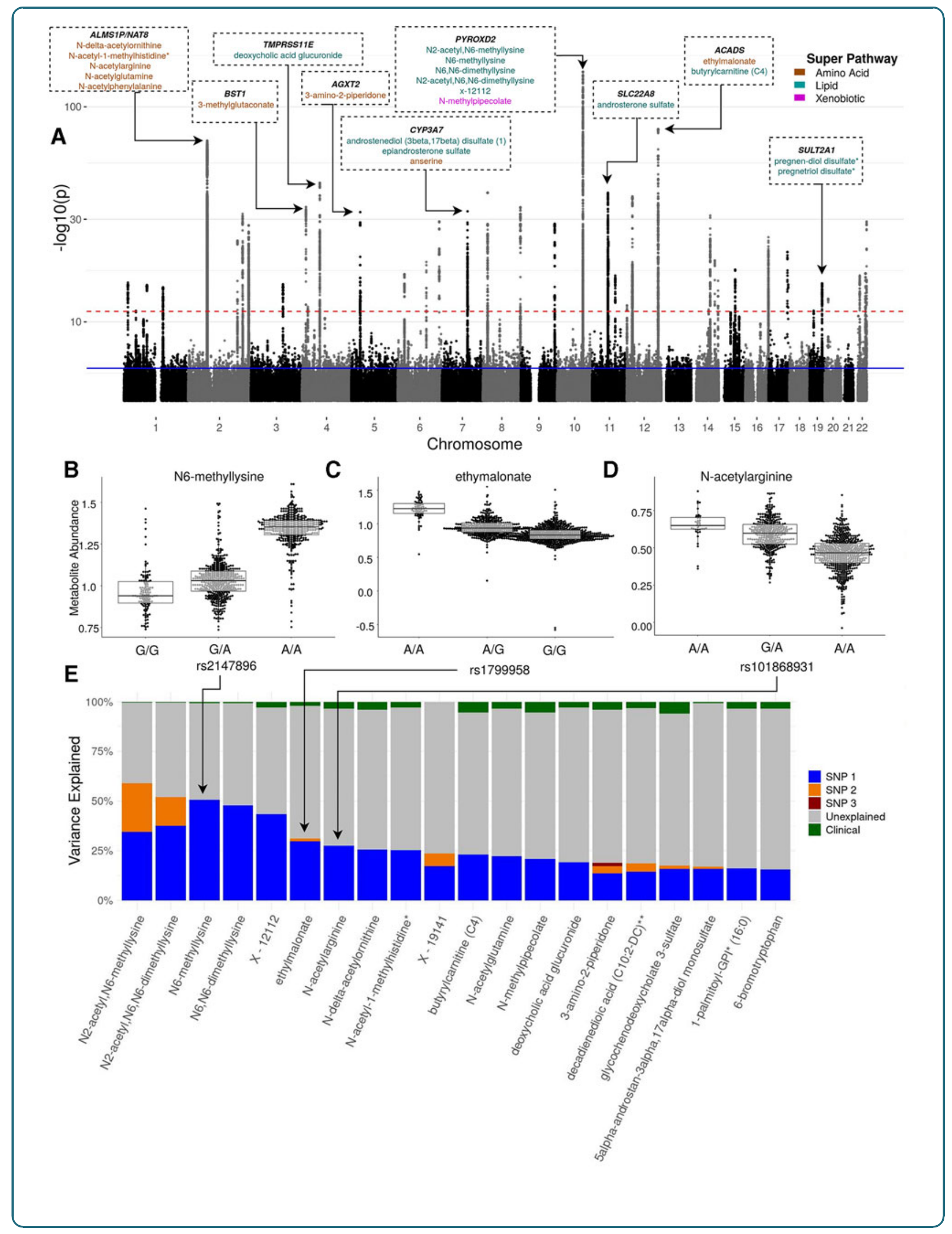




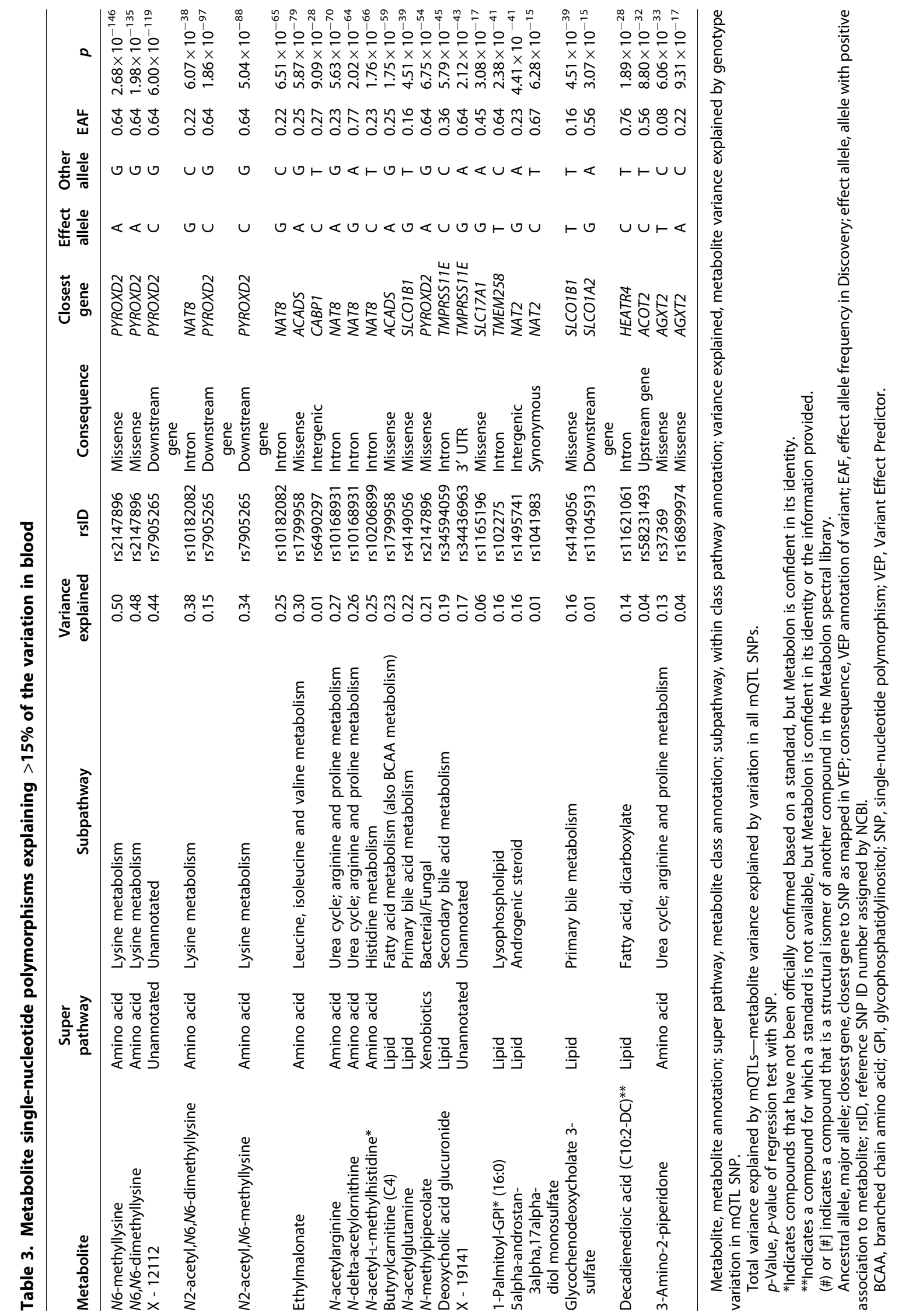



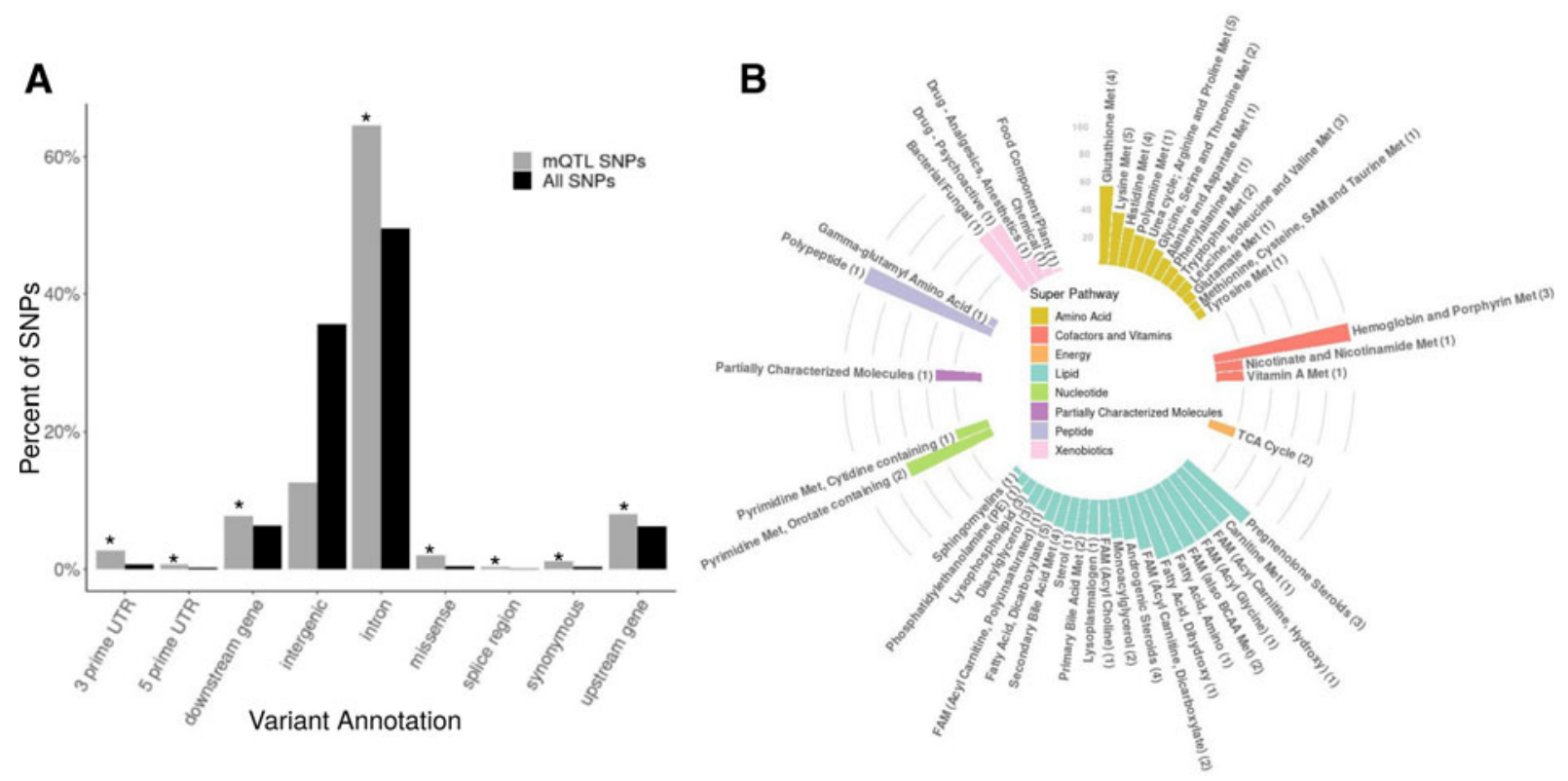

FIG. 3. mQTL SNP enrichment analyses. (A) A bar plot showing the percentage by variant annotation of mQTL SNPs (black) and all SNPs tested (black). *Variant annotations significantly enriched in mQTLs. (B) A circular bar plot showing the percentage of each subpathway with at least one independent mQTL. The bars are colored by super pathway and labeled by subpathway, with the total number of metabolites in the subpathway with an MQTL in parentheses. FAM, fatty acid metabolism; Met, metabolism.

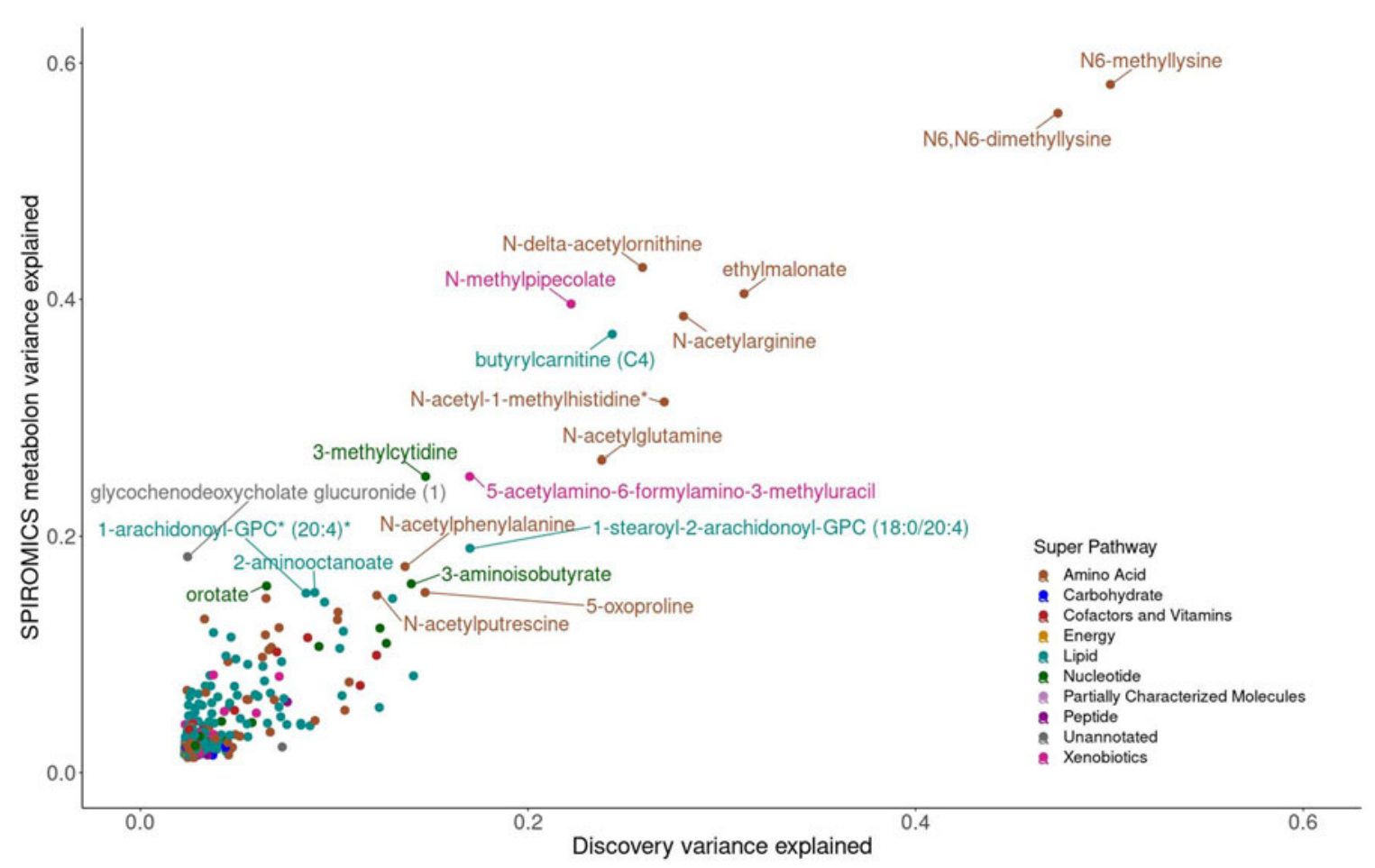

FIG. 4. Scatter plot of metabolite variance explained by lead SNP in Discovery and SPIROMICS-Metabolon cohorts. We calculated the variance explained $\left(r^{2}\right)$ for each lead mQTL SNP and metabolite by cohort. Metabolite colors represent super pathway annotation and are labeled if variance explained by genotype was $>0.15$ in either cohort. 


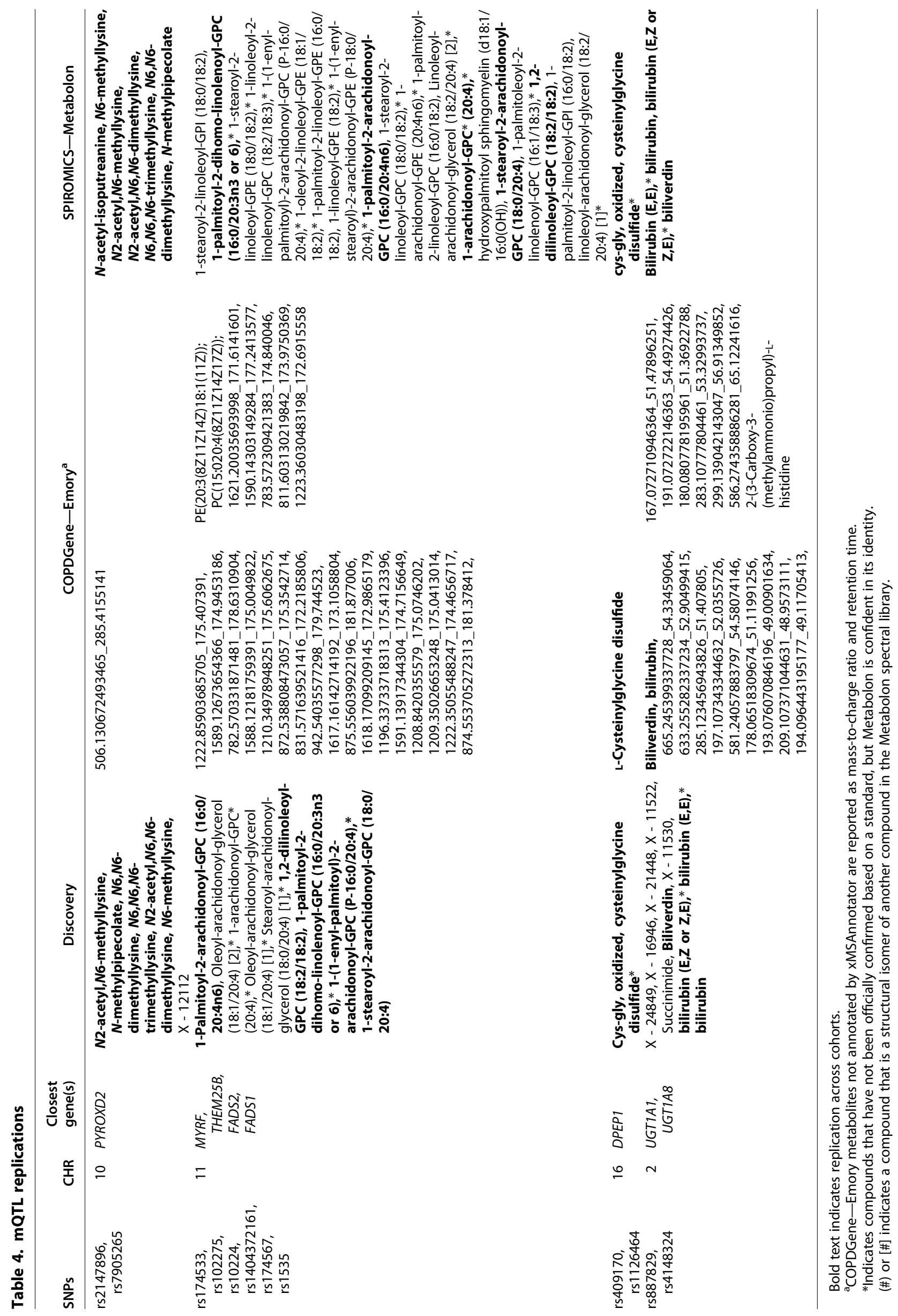


Fig. S6), with similar metabolomic variance explained. Replications were seen for several chromosomal regions across Discovery, COPDGene-Emory, and SPIROMICS-Metabolon, including lysine metabolites with SNPs in PYROXD2 on chromosome 10, phosphocholines with SNPs in MYRF, THEM25B, and FADS1/2 regions on chromosome 11, cysteinylglycine disulfide with SNPs in DPEP, and bilirubin/biliverdin with SNPs in the UGT1A1/8 regions on chromosome 2, with strong signals in the PYROXD2 of chromosome 10 , as well as the FADS1/FADS2 region of chromosome 11 (Table 3 and Supplementary Fig. S5).

Integration of genes, metabolites, and COPD phenotypes

The met-QTL analysis provides evidence of geneticmetabolite abundance links, but not specific to COPD. To identify affected genetic-metabolite-phenotype pathways in a strictly data-driven manner, we first created a GGM network of co-abundant metabolites and then used those results to identify modules associated with disease phenotypes. This method has been shown to enhance classical association analyses by increasing statistical power through aggregating metabolite abundance and recognizing disease-driven interplay between pathways. ${ }^{55}$ To infer the relationship between the genomics, metabolomics, and phenotypic data, the mWAS results were combined with phenotype-driven modules by adding edges between met-QTL SNPs and metabolites.

In the first step, given all 995 metabolites, a GGM was created. Then, nodes representing independent met-QTL SNPs were added, with edges linking to associated metabolites. The final GGM network was sparse, containing a combined 693 nodes (582 metabolite and 79 SNP nodes) and 505 significant, undirected edges between any node (metabolites or genes).

Then, in the second step, we used the MoDentfy module-identification algorithm with the COPD phenotypes to find phenotype-associated modules (i.e., subnetworks of the GGM associated with a specific phenotype). Testing all 5 COPD phenotypes separately, this resulted in 26 significant modules, sometimes associated with more than 1 phenotype, which included metabolites missed in univariate analysis (Fig. 5 and Supplementary Table S19). For example, a module of three lactosylceramides was associated with percent emphysema (adjusted $p$-value $=0.00018$ ) and a module of three hippurates was associated with $\mathrm{FEV}_{1} / \mathrm{FVC}$ (adjusted $p$-value $=0.04$ ), all of which had not been significantly associated previously (although the same direction of effect was observed between the modules and independent metabolites; see Supplementary Table S19). Other modules reconfirmed previously identified associations, like the module most associated with $\mathrm{FEV}_{1} \mathrm{pp}$ (Bonferroni adjusted $p$-value $=3 \times 10^{-7}$ ) containing the amino acid vanillylmandelate and two unannotated metabolites (X - 12707 and X - 13553).

Of the 26 modules associated with COPD phenotypes, 6 associated with airflow obstruction phenotypes had edges to gene nodes (Table 5). For these modules, we utilized the percent-variance-explained results to determine the genetic effect on individual metabolite abundances and the module, represented by the first PC of the module. We further compared the variance explained by genetic variance to the percent explained by clinical and environmental variance, as represented by the covariates used previously. While, as reported earlier, significant variance in individual metabolites was explained by genetic variance (ranging from 5\% to $18.9 \%$ ), the opposite effect was observed in COPD phenotype-associated modules, with the exception of the module of dicarboxylate fatty acids containing decadienedioic acid (C10:2-DC) ${ }^{\star *}$ and tetradecadienedioate $(\mathrm{C} 14: 2-\mathrm{DC})^{*}$ where $13.2 \%$ of the module variance was explained by variation in SNPs rs11626972 and rs58231493 and only $3 \%$ was explained by clinical and environmental variance.

\section{Discussion}

While COPD is a disease of the lungs, we find a strong systemic metabolomic signature in the blood even after adjusting for common risk factors such as smoking. This is consistent with observations that COPD is associated with extrapulmonary diseases such as cardiovascular disease, osteoporosis, muscle wasting, and insulin resistance. Many of the metabolomic signatures we identified are similar to those found in these diseases (e.g., sphingolipids in cardiovascular and metabolic disorders $^{64}$ or bone remodeling, ${ }^{65}$ acylcarnitines in osteoporosis, ${ }^{66}$ and diacylglycerols in insulin resistance ${ }^{67}$ ), suggesting common systemic pathways are important in COPD pathogenesis.

The lone metabolite associated with exacerbation frequency, of TMAP, further exemplifies the potential systemic effects of COPD. Although the reported association is novel in COPD, TMAP was recently identified as a biomarker of chronic kidney disease, ${ }^{68}$ a comorbidity of COPD ${ }^{69}$ with a similar inverse association between 


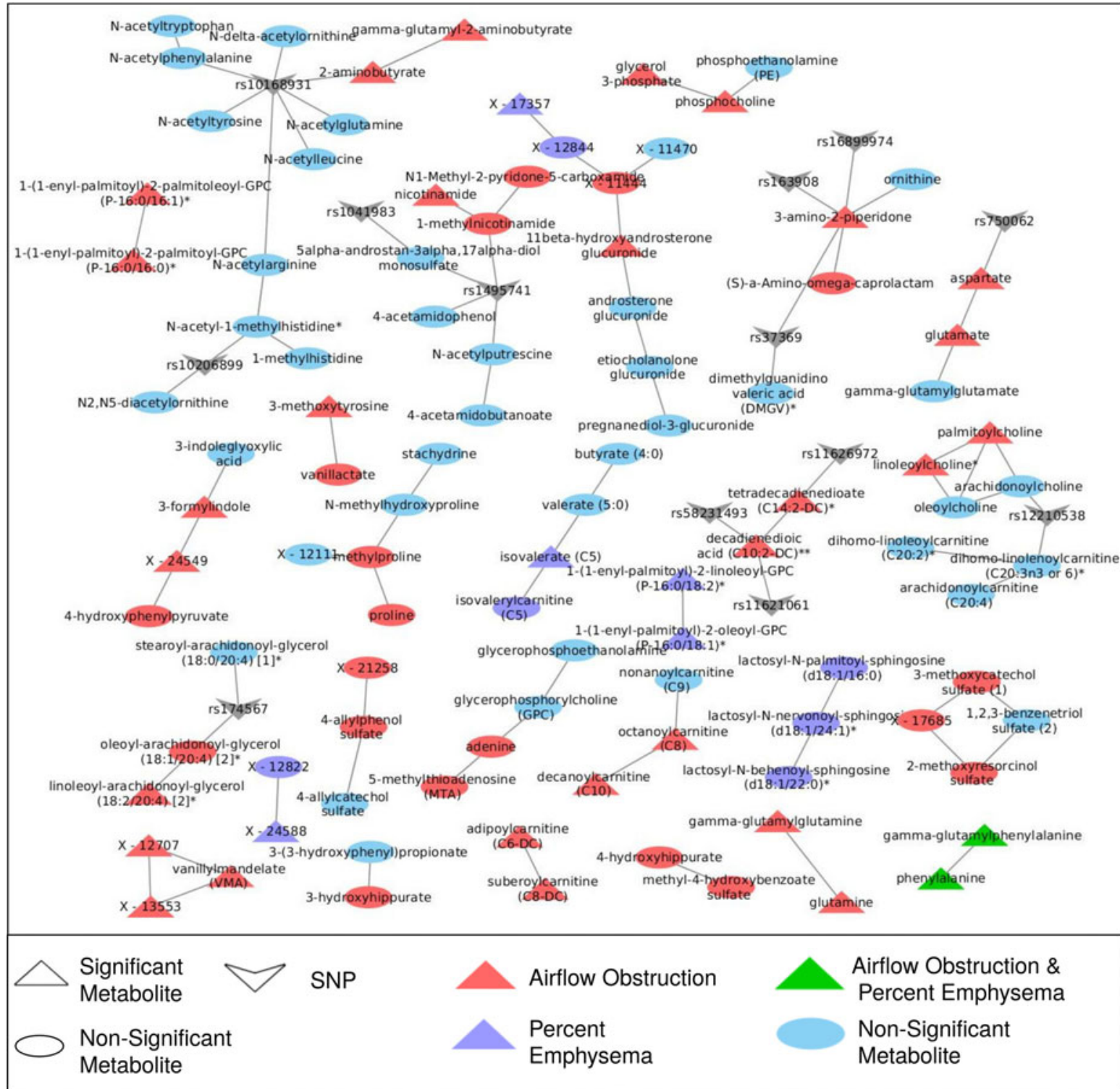

FIG. 5. Phenotype-driven modules. Cytoscape network representation of metabolite modules significantly associated one or more COPD phenotypes. Circular nodes are nonsignificant, while triangular nodes were significant in univariate analysis. "V" nodes mQTL SNPs. The color corresponds to the phenotype with which the metabolites in the module are associated; red indicates airflow obstruction phenotypes ( $F E V_{1} / F V C$ or $F E V_{1} p p$ ), purple indicates percent emphysema, green indicates both spirometric phenotypes and percent emphysema, and blue are nonsignificant metabolites.

TMAP abundances and disease severity. Moreover, while the biologic origin of TMAP has not yet been identified, it is suggested that myosin light-chain (MLC) protein degradation results in the release of TMAP. ${ }^{68}$ Disruption in MLC isoforms has been observed in COPD subjects with reduced activity and low oxygen supply, yet further work is needed to understand the pathophysiology of TMAP in exacerbations.

Systemic mitochondrial dysfunction, heightened in lungs with cigarette smoke-induced inflammatoryoxidative stress, has been implicated in the pathology of emphysema. ${ }^{7,70}$ We found further support of this 


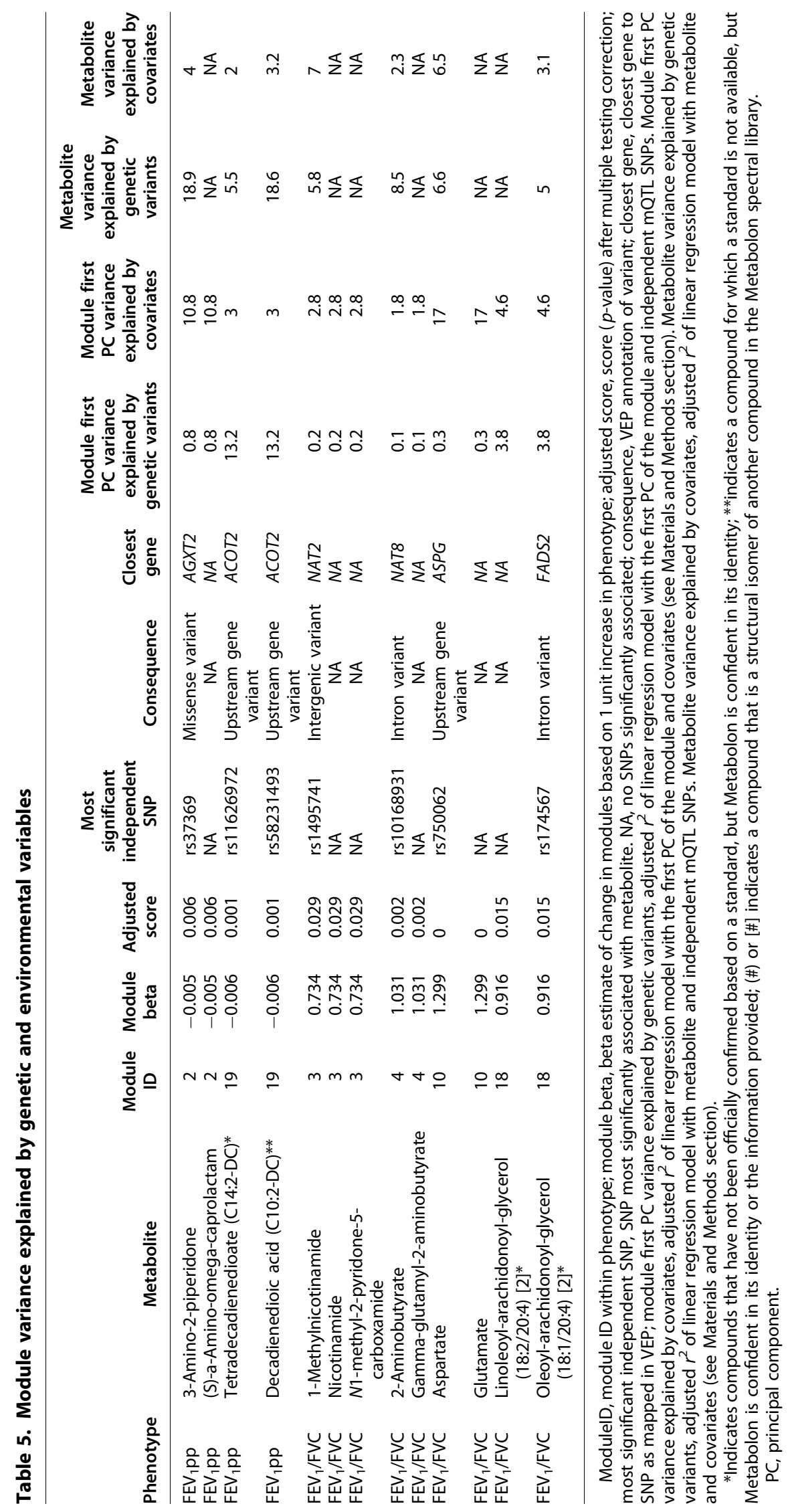


as citrate was uniquely associated with the percent emphysema phenotype in regression analyses, demonstrating potential TCA cycle dysregulation. The increased power of phenotype-driven GGM network analysis positively associated three lactosylceramides with percent emphysema.

Abnormalities in glycosphingolipid metabolism have been noted to be associated with COPD phenotypes. For example, there is evidence for correlation between glycobiosyl ceramides and COPD exacerbations, ${ }^{6}$ and that glucosyl ceramide synthase, which governs the first step in the glycosphingolipid metabolism, is an important determinant of cell fate of lung endothelial cells. ${ }^{71}$ Moreover, lactosylceramide accumulation was recently identified as a common pathogenic mechanism that induces apoptotic-inflammatory responses and aberrantautophagy leading to emphysema. ${ }^{72}$

Lactosylceramides can directly inhibit electron chain complexes, which enhance the production of reactive oxidation species in the mitochondria, potentially leading to lung inflammation and airway remodeling characteristic of emphysema. ${ }^{72,73}$ As the initial products in the formation of glycosphingolipids (e.g., lactosylceramides) are upregulated in insulin-resistant patients, increased lactosylceramide abundance may demonstrate comorbid mitochondrial dysregulation in COPD and metabolic disorders.

Several other metabolites previously associated with insulin resistance and other metabolic disorders were concordantly associated with COPD phenotypes. These included aromatic amino acids (phenylalanines) and BCAAs with both percent emphysema and airflow obstruction, as well as diacylglycerols, gamma-glutamyl amino acids, sphingomyelins, and lipids involved in the fatty acid and phospholipid metabolism, specifically with airflow obstruction. Abnormal amino acid and lipid metabolism may result from reduced dietary intake, oxidative stress, and increased strain on respiratory muscles with anoxia, leading to an active metabolic COPD state in COPD patients. ${ }^{74,75}$ These results confirm the findings of smaller studies that have shown strong associations between phospholipid-derived sphingolipids and COPD, ${ }^{6,76}$ and a recent two-cohort population study (KORA and ARIC) with 4347 controls and 393 COPD subjects that identified similar associations with BCAAs, aromatic amino acids, and glutamine/glutamate metabolites. ${ }^{8}$

However, our study differed from the KORA and ARIC study, in that we found more associations with $\mathrm{FEV}_{1} / \mathrm{FVC}$ than $\mathrm{FEV}_{1} \mathrm{pp}$, indicating a metabolic signa- ture of airflow obstruction. This may be because COPDGene primarily enrolled current and former smokers ( $>10$ and $>20$ pack-years, respectively), oversampled for COPD cases, was older, and included only NHW subjects (ARIC had many AA subjects). Although we adjusted for these variables in our analyses, age and smoking have strong influences on metabolome, and thus the generalizability might be limited.

While lifestyle behaviors (e.g., smoking) are important risk factors for COPD, there is evidence that as much as $37 \%$ of the variability in lung function is genetic. ${ }^{13}$ To explore this, we first sought to identify the genetic effect on the metabolome, detecting significant SNP-metabolite associations in 109 (11\%) of the metabolites tested (similar to the 119 of 529 [22\%] metabolites previously reported by Shin et al). ${ }^{54}$ The strongest association was between missense SNP rsrs2147896 in PYROXD2 and N6methyllysine $\left(p=3.97 \times 10^{-146}\right)$. PYROXD2 has been associated with lysine metabolites in other mWAS, including N6-methyllysine, as well as trimethylamine in urine and dimethylamine in plasma. ${ }^{77}$ Of the 79 independent loci identified with recursive conditioning, 47 novel SNPs were found, including rs58231493, an upstream variant of ACOT2 (coding for Acyl-CoA Thioesterase 2) associated with decadienedioic acid (C10:2-DC)**.

One of the most promising met-QTLs, as it was significant across replication cohorts, was in UGT1A region and associated with bilirubin pathway metabolites. In the Framingham Heart Study Offspring cohort, those with higher bilirubin due to a genetic polymorphism affecting the UGT1A1 enzyme of bilirubin metabolism (the enzyme defect that leads to Gilbert's syndrome) had one-third the risk of cardiovascular events compared to wild-type carriers with normal bilirubin concentrations. ${ }^{78}$ Higher levels of serum bilirubin have been inversely associated with the risk of COPD severity, progression, and mortality, ${ }^{79,80}$ and more recently, fewer COPD exacerbations. ${ }^{81}$ In in vitro and animal studies, bilirubin prevents oxidation of lipids, which may protect the COPD lung by inhibiting lipid peroxidation. ${ }^{80,82}$ Variability in bilirubin concentration has been previously associated with SNPs in UGT1A region. ${ }^{83,84}$ In our analysis, we found both bilirubin and biliverdin nearing the conservative Bonferroni significance threshold for associations with airflow obstruction phenotypes $(p=3.02 \times$ $10^{-3}$ and $6.83 \times 10^{-4}$ with $\mathrm{FEV}_{1} / \mathrm{FVC}$, respectively). Integrating the genetic associations suggests that the observed associations between bilirubin/biliverdin and COPD may be mediated through genetics. 
While there appears to be a strong genetic effect on the metabolome overall, there is less evidence for the genetic regulation of COPD-associated metabolites. Only 10 of the metabolites associated with COPD phenotypes through regression or phenotype-driven network analysis also had met-QTL SNP associations. Moreover, it was only within the module containing the fatty acids decadienedioic acid (C10:2-DC) ${ }^{\star *}$ and tetradecadienedioate $(14: 2-\mathrm{DC})^{\star}$ that more variance was explained by an upstream variant of ACOT2 than environmental variables.

The protein that ACOT2 codes for, Acyl-CoA thioesterase-2, has been shown to facilitate mitochondrial fatty acid oxidation in mouse models and may warrant further study. ${ }^{85}$ The lack of evidence for genetic regulation in the COPD metabolome may implicate other downstream regulation (e.g., methylation, post-translational modification, and metabolism of exogenous metabolites) having a greater effect. Thus, modifiable behaviors, like smoking, diet, and exercise, may have a greater effect on the COPD metabolism than genetic predisposition.

While this study was strengthened by the large number of subjects in a well-categorized cohort, there were several limitations. First, this analysis was performed using blood samples, as opposed to bronchial lavage fluid, which may better represent COPD phenotypes. ${ }^{33}$ It is well documented that the blood metabolome, across multiple metabolic pathways, is strongly affected by demographic factors, including age and sex, ${ }^{51,86}$ which we replicated in our initial exploratory analyses. COPD pathology begins in the lungs and then manifests as systemic dysregulation across several biologic tissues. However, the observed effects on the metabolome may still not be as pronounced within blood as the effects of age and sex.

Second, although this is one of the largest mWAS studies reported, 957 subjects are still small compared to clinical GWAS studies. The cohort was also restricted to NHW subjects, which limits generalizability over the entire population. Moreover, as many distinct and independent met-QTL SNPs were identified for many metabolites, there may be multiple mechanisms along genetic and metabolic pathways that influence observed metabolite intensities.

Another major challenge in metabolomics is crossplatform replication. Although we had two independent cohort metabolomics platforms available for replication and we identified similar met-QTLs across cohorts, the named metabolome features for these
met-QTL metabolites used three different annotation techniques (Metabolon was proprietary annotation; COPDGene-Emory used xMSannotator; and CU used Agilent MassHunter and IDBrowser). These annotation strategies are optimized to the platforms and cross-annotation was challenging.

Despite these challenges, we were able to use the presence of common met-QTLs as evidence to support a specific annotation; however, since all the platforms were untargeted, it was sometimes unclear which of the three annotations was the correct annotation. Finally, the sample sizes our COPDGene-Emory and replication cohorts, as well as their limited metabolite annotation, greatly limited our statistical power to detect replicating met-QTLs. Further work with targeted metabolomics studies could assist with these met-QTL associations.

In conclusion, this study found evidence in the blood metabolome for systemic dysregulation of metabolic pathways affecting COPD phenotypes in a diseased population. By further assessing the blood metabolome for genetic regulation, we reproduced several known associations and identified many novel met-QTL SNPs. Furthermore, we expanded and contextualized metabolite associations through COPD phenotypedriven module identification, integrating the genetic associations into the network view. While we found nongenetic factors to explain more variance in COPDassociated metabolites than genetic, further work is needed, potentially integrating the metabolome with other omics data types (e.g., epigenomics and proteomics), to elucidate and characterize dysregulated pathways in COPD pathogenesis.

\section{Authors' Contributions}

L.A.G., K.K., and R.P.B. conceived the study. L.A.G., K.A.P., B.D.H., M.H.C., Y.Z., K.K., and R.P.B. developed the methodological framework. B.D.H., M.H.C., C.C-Q., N.R., V.O., D.P.J., K.U., G.M., and R.P.B. collected the data. L.A.G., S.J., C.C-Q., E.H-S., K.U., and G.M. curated the data. LAG implemented the formal analysis and created the visualizations. The original draft was written by L.A.G., K.K., and R.P.B. Review and editing were performed by L.A.G., K.A.P., B.D.H., M.H.C., Y.Z., C.C-H., N.R., I.P., W.W.L., W.K.O., V.O., G.M., K.K., and R.P.B. This project was supervised by K.K. and R.P.B. All authors have reviewed and approved of the final article. This article has been submitted solely to the Systems Medicine journal and is not published, in press, or submitted elsewhere. 


\section{Acknowledgments}

COPDGene phase 3

Grant support and disclaimer. The project described was supported by award number U01 HL089897 and award number U01 HL089856 from the National Heart, Lung, and Blood Institute. The content is solely the responsibility of the authors and does not necessarily represent the official views of the National Heart, Lung, and Blood Institute or the National Institutes of Health.

COPD foundation funding. The COPDGene ${ }^{\circledR}$ project is also supported by the COPD Foundation through contributions made to an Industry Advisory Board comprising AstraZeneca, Boehringer-Ingelheim, Genentech, GlaxoSmithKline, Novartis, and Sunovion.

COPDGene investigators: core units. Administrative Center: James D. Crapo, MD (PI); Edwin K. Silverman, MD, PhD (PI); Barry J. Make, MD; and Elizabeth A. Regan, MD, PhD.

Genetic Analysis Center: Terri Beaty, PhD; Ferdouse Begum, PhD; Peter J. Castaldi, MD, MSc; Michael Cho, MD; Dawn L. DeMeo, MD, MPH; Adel R. Boueiz, MD; Marilyn G. Foreman, MD, MS; Eitan HalperStromberg; Lystra P. Hayden, MD, MMSc; Craig P. Hersh, MD, MPH; Jacqueline Hetmanski, MS, $\mathrm{MPH}$; Brian D. Hobbs, MD; John E. Hokanson, $\mathrm{MPH}, \mathrm{PhD}$; Nan Laird, PhD; Christoph Lange, PhD; Sharon M. Lutz, PhD; Merry-Lynn McDonald, PhD; Margaret M. Parker, PhD; Dmitry Prokopenko, $\mathrm{PhD}$; Dandi Qiao, PhD; Elizabeth A. Regan, $\mathrm{MD}, \mathrm{PhD}$; Phuwanat Sakornsakolpat, MD; Edwin K. Silverman, MD, PhD; Emily S. Wan, MD; and Sungho Won, PhD.

Imaging Center: Juan Pablo Centeno; Jean-Paul Charbonnier, PhD; Harvey O. Coxson, PhD; Craig J. Galban, PhD; MeiLan K. Han, MD, MS; Eric A. Hoffman, Stephen Humphries, PhD; Francine L. Jacobson, MD, MPH; Philip F. Judy, PhD; Ella A. Kazerooni, MD; Alex Kluiber; David A. Lynch, MB; Pietro Nardelli, PhD; John D. Newell, Jr., MD; Aleena Notary; Andrea Oh, MD; Elizabeth A. Regan, MD, PhD; James C. Ross, $\mathrm{PhD}$; Raul San Jose Estepar, PhD; Joyce Schroeder, MD; Jered Sieren; Berend C. Stoel, PhD; Juerg Tschirren, $\mathrm{PhD}$; Edwin Van Beek, $\mathrm{MD}, \mathrm{PhD}$; Bram van Ginneken, PhD; Eva van Rikxoort, PhD; Gonzalo Vegas Sanchez-Ferrero, PhD; Lucas Veitel; George R. Washko, MD; and Carla G. Wilson, MS.

PFT QA Center, Salt Lake City, UT: Robert Jensen, $\mathrm{PhD}$.
Data Coordinating Center and Biostatistics, National Jewish Health, Denver, CO: Douglas Everett, $\mathrm{PhD}$; Jim Crooks, $\mathrm{PhD}$; Katherine Pratte, $\mathrm{PhD}$; Matt Strand, PhD; and Carla G. Wilson, MS.

Epidemiology Core, University of Colorado Anschutz Medical Campus, Aurora, CO: John E. Hokanson, $\mathrm{MPH}, \mathrm{PhD}$; Gregory Kinney, $\mathrm{MPH}, \mathrm{PhD}$; Sharon M. Lutz, PhD; and Kendra A. Young, PhD.

Mortality Adjudication Core: Surya P. Bhatt, MD; Jessica Bon, MD; Alejandro A. Diaz, MD, MPH; MeiLan K. Han, MD, MS; Barry Make, MD; Susan Murray, ScD; Elizabeth Regan, MD; Xavier Soler, MD; and Carla G. Wilson, MS.

Biomarker Core: Russell P. Bowler, MD, PhD; Katerina Kechris, $\mathrm{PhD}$; and Farnoush Banaei-Kashani, $\mathrm{PhD}$.

COPDGene investigators: clinical centers

Ann Arbor VA: Jeffrey L. Curtis, MD; Perry G. Pernicano, MD.

Baylor College of Medicine, Houston, TX: Nicola Hanania, MD, MS; Mustafa Atik, MD; Aladin Boriek, $\mathrm{PhD}$; Kalpatha Guntupalli, MD; Elizabeth Guy, MD; and Amit Parulekar, MD.

Brigham and Women's Hospital, Boston, MA: Dawn L. DeMeo, MD, MPH; Alejandro A. Diaz, MD, MPH; Lystra P. Hayden, MD; Brian D. Hobbs, MD; Craig Hersh, MD, MPH; Francine L. Jacobson, MD, MPH; and George Washko, MD.

Columbia University, New York, NY: R. Graham Barr, MD, DrPH; John Austin, MD; Belinda D’Souza, $\mathrm{MD}$; and Byron Thomashow, MD.

Duke University Medical Center, Durham, NC: Neil MacIntyre, Jr., MD; H. Page McAdams, MD; and Lacey Washington, MD.

HealthPartners Research Institute, Minneapolis, MN: Charlene McEvoy, MD, MPH, and Joseph Tashjian, MD.

Johns Hopkins University, Baltimore, MD: Robert Wise, MD; Robert Brown, MD; Nadia N. Hansel, $\mathrm{MD}, \mathrm{MPH}$; Karen Horton, MD; Allison Lambert, MD, MHS; and Nirupama Putcha, MD, MHS.

Lundquist Institute for Biomedical Innovationat Harbor UCLA Medical Center, Torrance, CA: Richard Casaburi, PhD, MD; Alessandra Adami, PhD; Matthew Budoff, MD; Hans Fischer, MD; Janos Porszasz, MD, $\mathrm{PhD}$; Harry Rossiter, $\mathrm{PhD}$; and William Stringer, MD.

Michael E. DeBakey VAMC, Houston, TX: Amir Sharafkhaneh, $\mathrm{MD}, \mathrm{PhD}$, and Charlie Lan, DO.

Minneapolis VA: Christine Wendt, MD; Brian Bell, MD; and Ken M. Kunisaki, MD, MS. 
Morehouse School of Medicine, Atlanta, GA: Marilyn G. Foreman, MD, MS; Eugene Berkowitz, MD, $\mathrm{PhD}$; and Gloria Westney, MD, MS.

National Jewish Health, Denver, CO: Russell Bowler, $\mathrm{MD}, \mathrm{PhD}$, and David A. Lynch, MB.

Reliant Medical Group, Worcester, MA: Richard Rosiello, MD, and David Pace, MD.

Temple University, Philadelphia, PA: Gerard Criner, MD; David Ciccolella, MD; Francis Cordova, MD; Chandra Dass, MD; Gilbert D’Alonzo, DO; Parag Desai, MD; Michael Jacobs, PharmD; Steven Kelsen, $\mathrm{MD}, \mathrm{PhD}$; Victor Kim, MD; A. James Mamary, MD; Nathaniel Marchetti, DO; Aditi Satti, MD; Kartik Shenoy, MD; Robert M. Steiner, MD; Alex Swift, MD; Irene Swift, MD; and Maria Elena Vega-Sanchez, MD.

University of Alabama, Birmingham, AL: Mark Dransfield, MD; William Bailey, MD; Surya P. Bhatt, $\mathrm{MD}$; Anand Iyer, MD; Hrudaya Nath, MD; and J. Michael Wells, MD.

University of California, San Diego, CA: Douglas Conrad, MD; Xavier Soler, MD, PhD; and Andrew Yen, MD.

University of Iowa, Iowa City, IA: Alejandro P. Comellas, MD; Karin F. Hoth, PhD; John Newell, Jr., MD; and Brad Thompson, MD.

University of Michigan, Ann Arbor, MI: MeiLan K. Han, MD MS; Ella Kazerooni, MD MS; Wassim Labaki, MD MS; Craig Galban, $\mathrm{PhD}$; and Dharshan Vummidi, MD.

University of Minnesota, Minneapolis, MN: Joanne Billings, MD; Abbie Begnaud, MD; and Tadashi Allen, MD.

University of Pittsburgh, Pittsburgh, PA: Frank Sciurba, MD; Jessica Bon, MD; Divay Chandra, MD, MSc; Carl Fuhrman, MD; and Joel Weissfeld, MD, MPH.

University of Texas Health, San Antonio, San Antonio, TX: Antonio Anzueto, MD; Sandra Adams, MD; Diego Maselli-Caceres, MD; Mario E. Ruiz, MD; and Harjinder Singh.

The authors thank the SPIROMICS participants and participating physicians, investigators, and staff for making this research possible. The authors thank the SPIROMICS participants and participating physicians, investigators, and staff for making this research possible. More information about the study and how to access SPIROMICS data is at www.spiromics.org. We would like to acknowledge the following current and former investigators of the SPIROMICS sites and reading centers: Neil E. Alexis, MD; Wayne $\mathrm{H}$. Anderson, PhD; Mehrdad Arjomandi, MD; Igor Barjaktarevic, MD, PhD; R. Graham Barr, MD, DrPH; Lori A. Bateman, MSc; Surya P. Bhatt, MD; Eugene R.
Bleecker, MD; Richard C. Boucher, MD; Russell P. Bowler, MD, PhD; Stephanie A. Christenson, MD; Alejandro P. Comellas, MD; Christopher B. Cooper, $\mathrm{MD}, \mathrm{PhD}$; David J. Couper, $\mathrm{PhD}$; Gerard J. Criner, MD; Ronald G. Crystal, MD; Jeffrey L. Curtis, MD; Claire M. Doerschuk, MD; Mark T. Dransfield, MD; Brad Drummond, MD; Christine M. Freeman, PhD; Craig Galban, PhD; MeiLan K. Han, MD, MS; Nadia N. Hansel, MD, MPH; Annette T. Hastie, PhD; Eric A. Hoffman, PhD; Yvonne Huang, MD; Robert J. Kaner, MD; Richard E. Kanner, MD; Eric C. Kleerup, MD; Jerry A. Krishnan, MD, PhD; Lisa M. LaVange, PhD; Stephen C. Lazarus, MD; Fernando J. Martinez, MD, MS; Deborah A. Meyers, PhD; Wendy C. Moore, MD; John D. Newell Jr., MD; Robert Paine, III, MD; Laura Paulin, MD, MHS; Stephen P. Peters, MD, PhD; Cheryl Pirozzi, MD; Nirupama Putcha, MD, MHS; Elizabeth C. Oelsner, MD, MPH; Wanda K. O’Neal, PhD; Victor E. Ortega, MD, PhD; Sanjeev Raman, MBBS, MD; Stephen I. Rennard, MD; Donald P. Tashkin, MD; J. Michael Wells, MD; Robert A. Wise, MD; and Prescott G. Woodruff, MD, MPH. The project officers from the Lung Division of the National Heart, Lung, and Blood Institute were Lisa Postow, PhD, and Lisa Viviano, BSN; SPIROMICS was supported by contracts from the NIH/NHLBI (HHSN268200900013C, HHSN268200900014C, HHSN268200900015C, HHSN268200900016C, HHSN268200900017C, HHSN268200900018C, HHSN268200900019C, and HHSN268200900020C), grants from the NIH/NHLBI (U01 HL137880 and U24 HL141762), and supplemented by contributions made through the Foundation for the NIH and the COPD Foundation from AstraZeneca/MedImmune; Bayer; Bellerophon Therapeutics; Boehringer-Ingelheim Pharmaceuticals, Inc.; Chiesi Farmaceutici S.p.A.; Forest Research Institute, Inc.; GlaxoSmithKline; Grifols Therapeutics, Inc.; Ikaria, Inc.; Novartis Pharmaceuticals Corporation; Nycomed $\mathrm{GmbH}$; ProterixBio; Regeneron Pharmaceuticals, Inc.; Sanofi; Sunovion; Takeda Pharmaceutical Company; and Theravance Biopharma and Mylan.

\section{Author Disclosure Statement}

No competing financial interests exist.

\section{Funding Information}

This study was supported by National Heart, Lung, and Blood Institute (NHLBI R21 HL140376, R01HL129937, R01HL 095432, R01 HL089856, and R01 HL089897); and UL1 RR025680 from NCRR/HIH. 
Michael H. Cho was supported by R01 HL135142 and R01 HL137927.

Michael H. Cho has received grant support from GSK and Bayer and received speaking or consulting fees from Illumina and AstraZeneca.

Brian D. Hobbs is supported by NIH K08 HL136928 and the Parker B. Francis Research Opportunity Award. Katerina J. Kechis is supported by U01 CA235488, R01 HL152735, and R01 HL137995.

\section{Supplementary Material}

Supplementary Figure S1

Supplementary Figure S2 Supplementary Figure S3 Supplementary Figure S4 Supplementary Figure S5 Supplementary Figure S6 Supplementary Table S1 Supplementary Table S2 Supplementary Table S3 Supplementary Table S4 Supplementary Table S5 Supplementary Table S6 Supplementary Table S7 Supplementary Table S8 Supplementary Table S9 Supplementary Table S10 Supplementary Table S11 Supplementary Table S12 Supplementary Table S13 Supplementary Table S14 Supplementary Table S15 Supplementary Table S16 Supplementary Table S17 Supplementary Table S18 Supplementary Table S19

\section{References}

1. Long T, Hicks $M, Y u H C$, et al. Whole-genome sequencing identifies common-to-rare variants associated with human blood metabolites. Nat Genet. 2017:49:568-578.

2. Fiehn O. Metabolomics - the Link between Genotypes and Phenotypes. Plant Mol Biol 2002;48:155-171.

3. Ubhi BK, Riley JH, Shaw PA, et al. Metabolic profiling detects biomarkers of protein degradation in COPD patients. Eur Respir J. 2012;40:345355.

4. Ubhi BK, Cheng KK, Dong J, et al. Targeted metabolomics identifies perturbations in amino acid metabolism that sub-classify patients with COPD. Mol Biosyst. 2012;8:3125-3133.

5. Chen Q, Deeb RS, Ma Y, et al. Serum metabolite biomarkers discriminate healthy smokers from COPD smokers. PLoS One. 2015;10: e0143937.

6. Bowler RP, Jacobson S, Cruickshank C, et al. Plasma sphingolipids associated with chronic obstructive pulmonary disease phenotypes. Am J Respir Crit Care Med. 2015;191:275-284.
7. Cruickshank-Quinn $\mathrm{Cl}$, Jacobson $\mathrm{S}$, Hughes $\mathrm{G}$, et al. Metabolomics and transcriptomics pathway approach reveals outcome-specific perturbations in COPD. Sci Rep. 2018;8:17132.

8. Yu B, Flexeder C, McGarrah RW, 3rd, et al. Metabolomics identifies novel blood biomarkers of pulmonary function and COPD in the general population. Metabolites. 2019;9:61.

9. Johnson $\mathrm{CH}$, Ivanisevic J, Siuzdak G. Metabolomics: beyond biomarkers and towards mechanisms. Nat Rev Mol Cell Biol. 2016;17:451459.

10. Wishart DS, Mandal R, Stanislaus A, et al. Cancer Metabolomics and the human metabolome database. Metabolites. 2016:6:10.

11. World Health Organization. The top 10 causes of death 2018 [updated May 1, 2019]. https://www.who.int/news-room/fact-sheets/detail/thetop-10-causes-of-death last accessed December 1, 2019.

12. Burney PG, Patel J, Newson R, et al. Global and regional trends in COPD mortality, 1990-2010. Eur Respir J. 2015;45:1239-1247.

13. Zhou JJ, Cho MH, Castaldi PJ, et al. Heritability of chronic obstructive pulmonary disease and related phenotypes in smokers. Am J Respir Crit Care Med. 2013;188:941-947.

14. Friedlander $A L$, Lynch $D$, Dyar $L A$, et al. Phenotypes of chronic obstructive pulmonary disease. COPD. 2007;4:355-384.

15. Vogelmeier CF, Criner GJ, Martinez FJ, et al. Global strategy for the diagnosis, management, and prevention of chronic obstructive lung disease 2017 report. GOLD executive summary. Am J Respir Crit Care Med. 2017;195:557-582.

16. Chatila WM, Thomashow BM, Minai OA, et al. Comorbidities in chronic obstructive pulmonary disease. Proc Am Thorac Soc. 2008;5:549555.

17. Rotival M, Zeller T, Wild PS, et al. Integrating genome-wide genetic variations and monocyte expression data reveals trans-regulated gene modules in humans. PLoS Genet. 2011;7:e1002367.

18. Sun BB, Maranville JC, Peters JE, et al. Genomic atlas of the human plasma proteome. Nature. 2018;558:73-79.

19. Rhee EP, Ho JE, Chen $\mathrm{MH}$, et al. A genome-wide association study of the human metabolome in a community-based cohort. Cell Metab. 2013;18: 130-143.

20. Hobbs BD, de Jong K, Lamontagne M, et al. Genetic loci associated with chronic obstructive pulmonary disease overlap with loci for lung function and pulmonary fibrosis. Nat Genet. 2017;49:426-432

21. Regan EA, Hokanson JE, Murphy JR, et al. Genetic epidemiology of COPD (COPDGene) study design. COPD. 2010;7:32-43.

22. Couper D, LaVange LM, Han M, et al. Design of the Subpopulations and Intermediate Outcomes in COPD Study (SPIROMICS). Thorax. 2014;69: 491-494.

23. Wells JM, Arenberg DA, Barjaktarevic I, et al. Safety and tolerability of comprehensive research bronchoscopy in COPD: results from the SPIROMICS Bronchoscopy sub-study. Ann Am Thorac Soc. 2019;16: 439-446.

24. Wan ES, Castaldi PJ, Cho MH, et al. Epidemiology, genetics, and subtyping of preserved ratio impaired spirometry (PRISm) in COPDGene. Respir Res. 2014;15:89.

25. Lynch DA, Moore CM, Wilson C, et al. CT-based visual classification of emphysema: association with mortality in the COPDGene study. Radiology. 2018;288:859-866.

26. Bowler RP, Kim V, Regan E, et al. Prediction of acute respiratory disease in current and former smokers with and without COPD. Chest. 2014;146: 941-950.

27. Evans AM, DeHaven CD, Barrett T, et al. Integrated, nontargeted ultrahigh performance liquid chromatography/electrospray ionization tandem mass spectrometry platform for the identification and relative quantification of the small-molecule complement of biological systems. Anal Chem. 2009;81:6656-6667.

28. Dehaven CD, Evans AM, Dai $H$, Lawton KA. Organization of GC/MS and LC/MS metabolomics data into chemical libraries. J Cheminform. 2010;2:9.

29. Miller MJ, Kennedy AD, Eckhart AD, et al. Untargeted metabolomic analysis for the clinical screening of inborn errors of metabolism. J Inherit Metab Dis. 2015;38:1029-1039.

30. Liu KH, Walker DI, Uppal K, et al. High-resolution metabolomics assessment of military personnel: evaluating analytical strategies for chemica detection. J Occup Environ Med. 2016;58(Suppl 1):S53-S61. 
31. Uppal K, Soltow QA, Strobel FH, et al. xMSanalyzer: automated pipeline for improved feature detection and downstream analysis of large-scale, non-targeted metabolomics data. BMC Bioinformatics. 2013;14:15.

32. Uppal K, Walker DI, Jones DP. xMSannotator: an R package for networkbased annotation of high-resolution Metabolomics Data. Anal Chem. 2017:89:1063-1067.

33. Halper-Stromberg E, Gillenwater L, Cruickshank-Quinn C, et al. Bronchoalveolar lavage fluid from COPD patients reveals more compounds associated with disease than matched plasma. Metabolites. 2019;9:157.

34. Cruickshank-Quinn C, Zheng LK, Quinn K, et al. Impact of blood collection tubes and sample handling time on serum and plasma metabolome and lipidome. Metabolites. 2018;8:88.

35. Cho MH, McDonald ML, Zhou X, et al. Risk loci for chronic obstructive pulmonary disease: a genome-wide association study and meta-analysis. Lancet Respir Med. 2014;2:214-225.

36. Sun W, Kechris $\mathrm{K}$, Jacobson $\mathrm{S}$, et al. Common genetic polymorphisms influence blood biomarker measurements in COPD. PLoS Genet. 2016;12: e1006011.

37. Cho MH, Castaldi PJ, Wan ES, et al. A genome-wide association study of COPD identifies a susceptibility locus on chromosome 19q13. Hum Mol Genet. 2012;21:947-957.

38. Li X, Ortega VE, Ampleford EJ, et al. Genome-wide association study of lung function and clinical implication in heavy smokers. BMC Med Genet. 2018;19:134.

39. Bijlsma S, Bobeldijk I, Verheij ER, et al. Large-scale human metabolomics studies: a strategy for data (pre-) processing and COPDGene-Emory. Anal Chem. 2006;78:567-574.

40. Hastie T, Tibshiran iR, Narasimhan B, et al. impute: impute: Imputation for Microarray Data. 1.56.0. 2018.

41. Wright FA, Sullivan PF, Brooks Al, et al. Heritability and genomics of gene expression in peripheral blood. Nat Genet. 2014;46:430-437.

42. Hughes G, Cruickshank-Quinn C, Reisdorph R, et al. MSPrep-summarization, normalization and diagnostics for processing of mass spectrometry-based metabolomic data. Bioinformatics. 2014;30: 133-134.

43. Oba S, Sato MA, Takemasa I, Monden M, et al. A Bayesian missing value estimation method for gene expression profile data. Bioinformatics. 2003 19:2088-2096.

44. Stacklies W, Redestig $\mathrm{H}$, Scholz $\mathrm{M}$, et al. pcaMethods-a bioconductor package providing PCA methods for incomplete data. Bioinformatics. 2007;23:1164-1167.

45. Johnson WE, Li C, Rabinovic A. Adjusting batch effects in microarray expression data using empirical Bayes methods. Biostatistics. 2007;8: 118-127.

46. Zemans RL, Jacobson S, Keene J, et al. Multiple biomarkers predict disease severity, progression and mortality in COPD. Respir Res. 2017. 18:117.

47. Shabalin AA. Matrix eQTL: ultra fast eQTL analysis via large matrix operations. Bioinformatics. 2012;28:1353-1358.

48. McLaren W, Gil L, Hunt SE, et al. The Ensembl variant effect predictor. Genome Biol. 2016;171):122.

49. Fisher RA. The logic of inductive inference. J R Statist Soc. 1935;98:39-82.

50. Benjamini $Y$, Hochberg $Y$. Controlling the false discovery rate: a practical and powerful approach to multiple testing. J R Statist Soc Series B Methodol. 1995:57:289-300.

51. Krumsiek J, Mittelstrass K, Do KT, et al. Gender-specific pathway differences in the human serum metabolome. Metabolomics. 2015;11:1815-1833.

52. Gagneur J, Jackson DB, Casari G. Hierarchical analysis of dependency in metabolic networks. Bioinformatics. 2003;19:1027-1034

53. Krumsiek J, Suhre K, Illig T, et al. Gaussian graphical modeling reconstructs pathway reactions from high-throughput metabolomics data. BMC Syst Biol. 2011;5:21.

54. Shin S-Y, Fauman EB, Petersen A-K, et al. An atlas of genetic influences on human blood metabolites. Nat Genet. 2014;46:543-550.

55. Do KT, Rasp DJN, Kastenmuller G, et al. MoDentify: phenotype-driven module identification in metabolomics networks at different resolutions. Bioinformatics. 2019;35:532-534.

56. Shannon P, Markiel A, Ozier O, et al. Cytoscape: a software environment for integrated models of biomolecular interaction networks. Genome Res. 2003;13:2498-2504
57. Shrine N, Guyatt AL, Erzurumluoglu AM, et al. New genetic signals for lung function highlight pathways and chronic obstructive pulmonary disease associations across multiple ancestries. Nat Genet. 2019;51: 481-493.

58. Sakornsakolpat $\mathrm{P}$, Prokopenko D, Lamontagne $\mathrm{M}$, et al. Genetic landscape of chronic obstructive pulmonary disease identifies heterogeneous cell-type and phenotype associations. Nat Genet. 2019;51: 494-505.

59. Buniello A, MacArthur JAL, Cerezo M, et al. The NHGRI-EBI GWAS Catalog of published genome-wide association studies, targeted arrays and summary statistics 2019. Nucleic Acids Res. 2019;47:D1005D1012.

60. Li Y, Sekula P, Wuttke M, et al. Genome-wide association studies of metabolites in patients with CKD identify multiple loci and illuminate tubular transport mechanisms. J Am Soc Nephrol. 2018:29:15131524.

61. Krumsiek J, Suhre K, Evans AM, et al. Mining the unknown: a systems approach to metabolite identification combining genetic and metabolic information. PLoS Genet. 2012;8:e1003005.

62. Rueedi R, Ledda M, Nicholls AW, et al. Genome-wide association study of metabolic traits reveals novel gene-metabolite-disease links. PLoS Genet. 2014;10:e1004132.

63. Lemaitre RN, Tanaka T, Tang W, et al. Genetic loci associated with plasma phospholipid n-3 fatty acids: a meta-analysis of genome-wide association studies from the CHARGE Consortium. PLoS Genet. 2011;7: e1002193.

64. Borodzicz S, Czarzasta K, Kuch M, et al. Sphingolipids in cardiovascular diseases and metabolic disorders. Lipids Health Dis. 2015 $14: 55$

65. Meshcheryakova A, Mechtcheriakova D, Pietschmann P. Sphingosine 1-phosphate signaling in bone remodeling: multifaceted roles and therapeutic potential. Expert Opin Ther Targets. 2017;21: 725-737.

66. Wang J, Yan D, Zhao A, et al. Discovery of potential biomarkers for osteoporosis using LC-MS/MS metabolomic methods. Osteoporos Int 2019:30:1491-1499.

67. Erion DM, Shulman Gl. Diacylglycerol-mediated insulin resistance. Nat Med. 2010;16:400-402.

68. Velenosi TJ, Thomson BKA, Tonial NC, et al. Untargeted metabolomics reveals $\mathrm{N}, \mathrm{N}, \mathrm{N}$-trimethyl-L-alanyl-L-proline betaine (TMAP) as a nove biomarker of kidney function. Sci Rep. 2019;9:6831.

69. Gaddam S, Gunukula SK, Lohr JW, et al. Prevalence of chronic kidney disease in patients with chronic obstructive pulmonary disease: a systematic review and meta-analysis. BMC Pulm Med. 2016 $16: 158$

70. Ran N, Pang Z, Gu Y, et al. An updated overview of metabolomic profile changes in chronic obstructive pulmonary disease. Metabolites. 2019;9: 111

71. Koike K, Berdyshev EV, Mikosz AM, et al. Role of glucosylceramide in lung endothelial cell fate and emphysema. Am J Respir Crit Care Med. 2019; 200:1113-1125.

72. Bodas M, Min T, Vij N. Lactosylceramide-accumulation in lipid-rafts mediate aberrant-autophagy, inflammation and apoptosis in cigarette smoke induced emphysema. Apoptosis. 2015;20:725-739.

73. Michaeloudes $\mathrm{C}$, Kuo CH, Haji G, et al. Metabolic re-patterning in COPD airway smooth muscle cells. Eur Respir J. 2017;50:1700202.

74. Ji Y, Wu Z, Dai Z, et al. Nutritional epigenetics with a focus on amino acids: implications for the development and treatment of metabolic syndrome. J Nutr Biochem. 2016;27:1-8.

75. Sergi G, Coin A, Marin S, et al. Body composition and resting energy expenditure in elderly male patients with chronic obstructive pulmonary disease. Respir Med. 2006;100:1918-1924.

76. Kilk K, Aug A, Ottas A, et al. Phenotyping of chronic obstructive pulmonary disease based on the integration of metabolomes and clinica characteristics. Int J Mol Sci. 2018;19:666.

77. Nicholson G, Rantalainen M, Li JV, et al. A genome-wide metabolic QTL analysis in Europeans implicates two loci shaped by recent positive selection. PLoS Genet. 2011;7:e1002270.

78. Lin JP, O'Donnell CJ, Schwaiger JP, et al. Association between the UGT1A $1 * 28$ allele, bilirubin levels, and coronary heart disease in the Framingham Heart Study. Circulation. 2006;114:1476-1481. 
79. Horsfall $L$, Rait $G$, Walters $K$, et al. Serum bilirubin and risk of respiratory disease and death. JAMA. 2011;305:691-697.

80. Apperley S, Park HY, Holmes DT, et al. Serum bilirubin and disease progression in mild COPD. Chest. 2015;148:169-175.

81. Brown KE, Sin DD, Voelker $\mathrm{H}$, et al. Serum bilirubin and the risk of chronic obstructive pulmonary disease exacerbations. Respir Res. 2017; 18:179.

82. Sedlak TW, Saleh M, Higginson DS, et al. Bilirubin and glutathione have complementary antioxidant and cytoprotective roles. Proc Natl Acad Sci U S A. 2009;106:5171-5176.

83. Benjamin EJ, Dupuis J, Larson MG, et al. Genome-wide association with select biomarker traits in the Framingham Heart Study. BMC Med Genet. 2007;8(Suppl 1):S11.

84. Milton JN, Sebastiani $\mathrm{P}$, Solovieff $\mathrm{N}$, et al. A genome-wide association study of total bilirubin and cholelithiasis risk in sickle cell anemia. PLoS One. 2012;7:e34741.

85. Moffat C, Bhatia L, Nguyen T, et al. Acyl-CoA thioesterase-2 facilitates mitochondrial fatty acid oxidation in the liver. J Lipid Res. 2014;55:24582470.

86. Darst BF, Koscik RL, Hogan KJ, et al. Longitudinal plasma metabolomics of aging and sex. Aging (Albany NY) 2019;11:1262-1282.

Cite this article as: Gillenwater $L A$, Pratte $K A$, Hobbs $B D$, Cho $M H$ Zhuang $Y$, Halper-Stromberg E, Cruickshank-Quinn C, Reisdorph N, Petrache I, Labaki WW, O'Neal WK, Ortega VE, Jones DP, Uppal K, Jacobson S, Michelotti G, Wendt CH, Kechris KJ, Bowler RP (2020) Plasma metabolomic signatures of chronic obstructive pulmonary disease and the impact of genetic variants on phenotype-driven modules, Network and Systems Medicine 3:1, 159-181, DOI: 10.1089/ nsm.2020.0009.

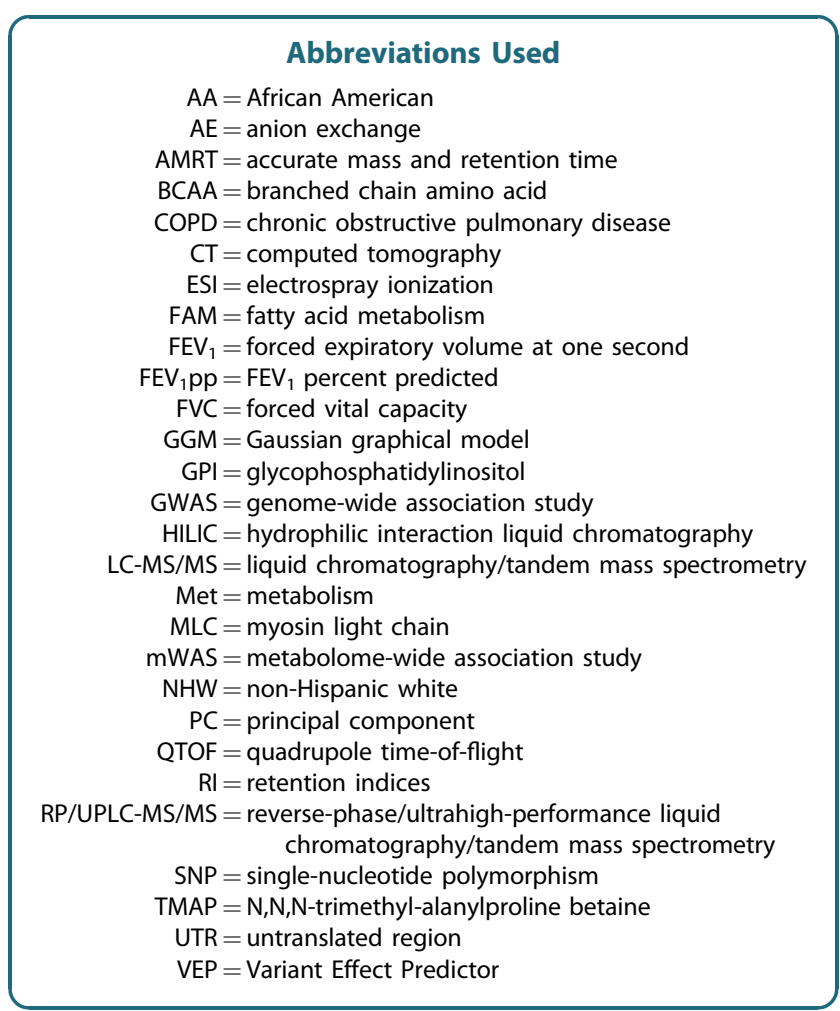

Publish in Network and Systems Medicine

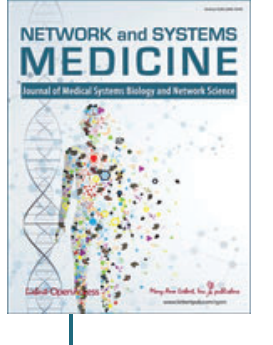

- Immediate, unrestricted online access

- Rigorous peer review

- Compliance with open access mandates

- Authors retain copyright

- Highly indexed

- Targeted email marketing

liebertpub.com/nsm 